\title{
Investigation of Extractable Organic Compounds in Deep-sea Hydrothermal Vent Fluids along the Mid-Atlantic Ridge
}

\author{
Thomas M. McCollom ${ }^{1}$, Jeffrey S. Seewald ${ }^{2}$, \& Christopher R. German ${ }^{2}$ \\ ${ }^{1}$ Laboratory for Atmospheric and Space Physics, Campus Box 600, University of Colorado, \\ Boulder CO 80309, email: mccollom@lasp.colorado.edu \\ ${ }^{2}$ Woods Hole Oceanographic Institution, Woods Hole, MA 02543
}

Abstract - The possibility that deep-sea hydrothermal vents may contain organic compounds produced by abiotic synthesis or by microbial communities living deep beneath the surface has led to numerous studies of the organic composition of vent fluids. Most of these studies have focused on methane and other light hydrocarbons, while the possible occurrence of more complex organic compounds in the fluids has remained largely unstudied. To address this issue, the presence of higher molecular weight organic compounds in deep-sea hydrothermal fluids was assessed at three sites along the Mid-Atlantic Ridge that span a range of temperatures (51 to $>360{ }^{\circ} \mathrm{C}$ ), fluid compositions, and host-rock lithologies (mafic to ultramafic). Sample were obtained at several sites within the Lucky Strike, Rainbow, and Lost City hydrothermal fields. Three methods were employed to extract organic compounds for analysis, including liquid:liquid extraction, cold trapping on the walls of a coil of titanium tubing, and pumping fluids through cartridges filled with solid phase extraction (SPE) sorbents. The only samples to consistently yield high amounts of extractable organic compounds were the warm (51-91 ${ }^{\circ} \mathrm{C}$ ), highly alkaline fluids from Lost City, which contained elevated concentrations of $\mathrm{C}_{8}, \mathrm{C}_{10}$, and $\mathrm{C}_{12} n$-alkanoic acids and, in some cases, trithiolane, hexadecanol, squalene, and cholesterol. Collectively, the $\mathrm{C}_{8}-\mathrm{C}_{12}$ acids can account for about $15 \%$ of the total dissolved organic carbon in the Lost City fluids. The even-carbon-number predominance of the alkanoic acids indicates a biological origin, but it is unclear whether these compounds are derived from microbial activity occurring within the hydrothermal chimney proximal to the site of fluid discharge or are transported from deeper within the system. Hydrothermal fluids from the Lucky Strike and Rainbow fields were characterized by an overall scarcity of extractable dissolved organic compounds. Trace amounts of aromatic hydrocarbons including phenanthrenes and benzothiophene were the only compounds that could be identified as indigenous components of these fluids. Although hydrocarbons and fatty acids were observed in some samples, those compounds were likely derived from particulate matter or biomass entrained during fluid collection. In addition, extracts of some fluid samples from the Rainbow field were found to contain an unresolved complex mixture (UCM) of organic compounds. This UCM shared some characteristics with organic matter extracted from bottom seawater, suggesting that the organic matter observed in these samples might represent seawater-derived compounds that had persisted, albeit with partial alteration, during circulation through the hydrothermal system. While there is considerable evidence that Rainbow and Lost City vent fluids contain methane and other light hydrocarbons produced through abiotic reduction of inorganic carbon, we found no evidence for more complex organic compounds with an abiotic origin in the same fluids. 


\section{INTRODUCTION}

Ever since deep-sea hydrothermal systems first began to be explored in the late 1970s, the occurrence of organic compounds in the hydrothermal fluids has been the subject of considerable scientific study. One of the primary motivations behind these studies has been to determine the source of the organic compounds and, in particular, to investigate whether some compounds might be generated by abiotic organic synthesis within the hydrothermal system (e.g., Welhan and Craig, 1983; Lilley et al., 1983, 1993; Berndt et al., 1996; Charlou et al., 2000, 2002, 2010; Holm and Charlou, 2001; McCollom and Seewald, 2001, 2007; Foustoukos and Seyfried, 2004; Proskurowski et al., 2008; Bradley and Summons, 2009; Lang et al., 2010, 2012). In addition, some organic compounds in deep-sea hydrothermal fluids may be produced by biological processes in subsurface environments, and can potentially provide insights into the activity of deep-seated microbial communities (Deming and Baross, 1993; Bradley and Summons, 2009; Reeves et al., 2014).

Field-based studies of organic compounds in deep-sea hydrothermal fluids have predominantly focused on the abundance and isotopic composition of methane and light hydrocarbons (e.g., Welhan and Craig, 1983; Lilley et al., 1983, 1993; Charlou et al., 1996, 2000, 2002: Proskurowski et al., 2008; Foustoukos et al., 2009). However, several studies have analyzed deep-sea hydrothermal fluids for the presence of other dissolved compounds including small carboxylic and amino acids (Haberstroh and Karl, 1989; Martens, 1990; Horiuchi et al., 2004; Lang et al., 2010, 2013; Klevenz et al., 2010; Fuchida et al., 2014). Relatively few studies have examined whether higher molecular weight hydrocarbons and other larger extractable organic compounds are present in deep-sea hydrothermal fluids (Brault et al., 1988; Holm and Charlou, 2001; Konn et al., 2009, 2012).

Sampling deep-sea hydrothermal fluids for higher hydrocarbons and other organic compounds presents significant challenges. Based on the abundance of volatile light hydrocarbons, higher molecular weight compounds might be expected to be present at nanomolar or lower concentrations, requiring them to be concentrated prior to analysis for most commonly used analytical methods. In addition, hydrocarbons and many other types of organic compounds must be extracted from the aqueous phase prior to analysis for conventional methods. While hydrocarbons and other low-polarity organic compounds have relatively high aqueous solubilities at the elevated temperatures and pressures that occur within many deep-sea hydrothermal systems, cooling of discharged fluids to ambient temperatures of the deep sea (usually $\sim 2{ }^{\circ} \mathrm{C}$ ) may cause these compounds to exsolve and precipitate on the surfaces of the sampling device. If only the fluid phase is analyzed, exsolved compounds may not be detected. Furthermore, organic compounds from other sources, including sweater and hydrothermal vent mineral deposits (e.g., Simoneit et al., 2004), can be inadvertently entrained into the sample during fluid collection. In addition, hydrothermal vent chimneys are often inhabited by biological communities, enhancing the potential for contamination from biomass if solids are incorporated during sample collection. Oils, hydraulic fluid, and other organic compounds leaking from the vehicle used to collect samples represent other potential sources of contamination.

This study was undertaken to investigate the occurrence of extractable organic compounds in hydrothermal systems along the Mid-Atlantic Ridge (MAR). Hydrothermal fluids were sampled at three sites along the MAR representing a range of conditions (Table 1). Hot (up to $366^{\circ} \mathrm{C}$ ), acidic fluids were sampled at systems hosted in ultramafic rocks at Rainbow and in basaltic rocks at Lucky Strike (Von Damm et al., 1998; Charlou et al. 2000, 2002). Warm ( 90 $\left.{ }^{\circ} \mathrm{C}\right)$, 
strongly alkaline fluids circulated through serpentinized ultramafic rocks were sampled at Lost City (Table 1) (Kelley et al., 2005). The Rainbow and Lost City were targeted for study because previous investigations have indicated that the methane and light hydrocarbons in the hydrothermal fluids at these sites may have an abiotic origin (Charlou et al., 2002, 2010; Proskurowski et al., 2008), and it has been suggested that other hydrocarbons with an abiotic origin might also be present (Holm and Charlou, 2001; Konn et al., 2012). None of the systems included in this study show any evidence for buried sediments that might contribute organic compounds to circulating hydrothermal fluids.

Concurrent with our study, Konn et al. $(2009,2012)$ also assessed the presence of extractable organic compounds in hydrothermal fluids from the Rainbow and Lost City sites, but used somewhat different methods for extraction and analysis. They reported detection of a number of extractable organic compounds in the fluids from both sites, including aliphatic, cyclic, and aromatic hydrocarbons as well as fatty acids. Previous work by Holm and Charlou (2001) had identified trace amounts of a homologous series of $\mathrm{C}_{16}-\mathrm{C}_{29} n$-alkanes in hydrothermal fluids at Rainbow. Other studies have identified formate, acetate, and amino acids in the fluids at Lost City (Lang et al., 2010, 2013). Reeves et al. (2014) report concentrations of methanethiol in hydrothermal fluids at many of the same sites included in this study, from samples collected on the same expedition as those reported here.

\section{METHODS}

109

110

111

112

113

114

115

116

117

118

119

120

121

122

123

124

125

126

127

128

129

130

131

132

133

134

\subsection{Fluid sampling}

Hydrothermal fluid samples were acquired in July 2008 using the Jason remotely operated vehicle (ROV) as part of the KNOX18RR expedition to the Mid-Atlantic Ridge on board the R/V Roger Revelle (Fig. 1). Hydrothermal fluids were sampled at several vent sites across the Lucky Strike, Rainbow, and Lost City hydrothermal fields, as summarized in Table 1. A single vent fluid sample was also obtained at the TAG hydrothermal site before a shipboard equipment failure prematurely terminated the cruise, but because no organic compounds were observed in the sample it is not discussed further here. At Lucky Strike and Rainbow, fluids were collected from a number of discrete vent locations distributed across the hydrothermal fields. Most samples were obtained at natural orifices in chimney structures where there was vigorous discharge of high-temperature $\left(>250{ }^{\circ} \mathrm{C}\right)$ hydrothermal fluids (e.g., Fig. 1a). For the Ecurie site at Rainbow, however, fluids were sampled from a cavity excavated into the side of a chimney structure that had moderately hot fluids $\left(\sim 60{ }^{\circ} \mathrm{C}\right)$ slowly venting from its apex (Fig. 1b). Theoretical studies have suggested that mixing zones within deep-sea hydrothermal systems might be particularly favorable environments for abiotic organic synthesis (Shock and Schulte, 1998), and this site was chosen because mixing of high temperature fluids with seawater is a likely reason for the low temperature of the vent fluids. Since the fluids venting at the apex of the Ecurie structure were too diffuse to sample, a cavity was excavated in the side of the chimney to gain access to more vigorous fluid flow (Fig. 1b). At Lost City, fluids were obtained at the Beehive site on the side of a large structure ( $\sim 60 \mathrm{~m}$ high) named Poseidon (Fig. 1c,d) and at an unnamed orifice at the top of the same structure. For comparison with the vent fluids, samples of deep seawater were also collected adjacent to the Lucky Strike, Lost City, and TAG sites using the same methods employed to collect hydrothermal fluids.

Because it was not clear at the outset what approach might be most effective for extraction of dissolved organic compounds from hydrothermal fluids, three different sampling strategies were employed. First, organic compounds were extracted from hydrothermal fluids acquired using 
isobaric gas-tight (IGT) syringe-type samplers (Seewald et al., 2002). A key feature of the IGT samplers is that the fluid intake rate can be regulated to minimize the amount of seawater entrained during sample collection. In an effort to minimize the contribution of organic compounds from background sources, the original design of the samplers was modified by replacement of the Viton O-rings on the sample chamber piston with Teflon O-rings to eliminate the need for a lubricant. Nevertheless, it was still necessary to use a Viton O-ring and a lubricant (Fluorolube) on the pressure seal for the sample chamber. Prior to each deployment, all wettable surfaces in the samplers were washed with successive rinses of methanol, dichloromethane (DCM), and hexane, and then dried in air. Despite these precautions, traces of fluorolube were found in some samples, presumably derived from the lubricant used on the pressure seal or from residual compounds in the sampler snorkels. The dead volume of the samplers (including the snorkel; $5 \mathrm{ml}$ ) was filled with deionized water (Fisher Scientific Optima, hereinafter referred to as Fisher $\mathrm{H}_{2} \mathrm{O}$ ) prior to deployment rather than the usual practice of filling this volume with bottom seawater. A Teflon cap was placed on the inlet of the sampler snorkel to limit seawater infiltration during transport to the seafloor.

In the second approach, hydrothermal fluids on the seafloor were pumped through a $4 \mathrm{~m}$ long coil of narrow titanium tubing (0.125" O.D. $\times 0.064 "$ I.D.) to extract organic compounds (this apparatus came to be known as the Peristaltic Organic Pump sampler or "POP gun”) (Fig. 2). The concept behind this device was that, because the Ti-coil is bathed in cold $\left(\sim 2{ }^{\circ} \mathrm{C}\right)$ seawater, cooling of the hydrothermal fluids as they passed through the tubing might induce precipitation of higher molecular weight organic compounds such as long-chain hydrocarbons and fatty acids on the tubing walls, owing to the decreasing solubility of these compounds with decreasing temperature and the large internal surface area of the narrow coiled tube. Prior to each deployment, the Ti tubing was washed with DCM followed by $\mathrm{MeOH}$, and then rinsed and filled with Fisher $\mathrm{H}_{2} \mathrm{O}$.

The third approach used to extract organic compounds was to attach a purpose-built cartridge containing a solid-phase extraction (SPE) sorbent to the outlet of the Ti tubing of the POP gun (Fig. 2 inset). The SPE cartridges were intended to extract additional organic compounds that remained dissolved in the fluid after passage through the Ti tubing. The SPE cartridges contained several grams of SPE microbeads within a $2.54 \mathrm{~cm}$ outside diameter Teflon tube (2.5 $\mathrm{cm}$ O.D. $\times 8 \mathrm{~cm}$ long), capped with porous Teflon frits to allow flow of fluids through the cartridge, and sealed with Swagelok fittings. Prior to deployment, the SPE sorbent was rinsed with $\sim 15 \mathrm{ml} \mathrm{MeOH}$ followed by $\sim 30 \mathrm{ml}$ of Fisher DI water while in the cartridge.

Two types of SPE sorbents targeted at different classes of organic compounds were used separately during deployments of the POP gun (Table 1). For most deployments, the "hydrophilic-lipophilic balanced" Oasis HLB sorbent (Waters Corporation) was used. This sorbent had been used previously for extraction of organic compounds from fluids collected at the Rainbow site (Holm and Charlou, 2001), and is designed to extract a broad spectrum of acidic, basic, and neutral organic compounds from aqueous fluids. At two sample locations (Rainbow and Lost City) fluids were extracted using the C8 phase from Grace Discovery Sciences. This phase is designed to target smaller non-polar organic compounds in the octane range, and was used in an attempt to isolate the type of alklylated monocyclic compounds that had been reported in previous SPE extracts of hydrothermal fluids (Konn et al., 2009, 2012).

When fully assembled on Jason, the POP gun sampler consisted of a coiled $4 \mathrm{~m}$ length of titanium tubing connected at the outlet end through Teflon tubing to the cartridge containing the SPE sorbent, and then to a peristaltic pump (Fig. 2). At the seafloor, the inlet of the POP gun was 
inserted into the orifice of a hydrothermal vent, and fluid was then pumped through the device for a period of about an hour in most cases. During sampling, flow of hydrothermal fluid through the POP gun was visually confirmed by the presence of shimmering (i.e., warm) fluid at the outlet of the peristaltic pump. A benchtop test with the fully assembled POP gun indicated that the flow rate was about $21 \mathrm{ml} \mathrm{min}^{-1}$, although this flow rate may not have been achieved by the peristaltic pump during operation at in situ pressures on the seafloor. Assuming the benchtop flow rate to be representative, it is estimated that $\sim 1.2 \mathrm{~L}$ of hydrothermal fluid would have been pumped through the POP gun during each 60 minute sample. Owing to logistical considerations, POP gun and SPE samples could not be obtained at every site where fluids were collected using IGT samplers during the cruise (Table 1).

\subsection{Sample processing}

The three sampling strategies employed required use of different protocols to extract organic compounds (Fig. 3). Following deployment and return to the surface, all sampling devices were immediately placed in a refrigerator at $4{ }^{\circ} \mathrm{C}$ until they were processed (usually within $\sim 2$ hours). All solvents used to extract organic compounds were Fisher Scientific Optima grade.

For the IGT samplers, the first two aliquots of fluid from each sample were collected in precleaned vials and stored at $-20^{\circ} \mathrm{C}$ for later analysis of inorganic chemistry (5 ml; Table 1) and dissolved free amino acids plus other organic compounds $(\sim 20 \mathrm{ml})$. The remaining fluid $(\sim 120$ $\mathrm{ml}$ ) was then transferred to a pre-cleaned glass bottle, where organic compounds were extracted by adding $\sim 8 \mathrm{ml}$ of dichloromethane (DCM) and then shaking vigorously for 10 minutes. The DCM phase was then removed by pipette into a separate vial for further processing. Because the Lost City hydrothermal fluids are strongly alkaline, a second liquid-liquid extraction was performed to extract any organic acid anions that may have been present following acidification to $\mathrm{pH}<3$ by addition of concentrated $\mathrm{HCl}$. The $\mathrm{pH}_{25^{\circ} \mathrm{C}}$ values of the Rainbow and Lucky Strike fluids were well below the $\mathrm{p} K_{\mathrm{a}}$ of alkanoic acids, so these samples were not acidified.

The DCM extracts were concentrated by evaporation under a gentle stream of $\mathrm{N}_{2}$ at room temperature. When the extract had been concentrated to $\sim 2 \mathrm{ml}$, it was capped and transferred to a freezer at $-20^{\circ} \mathrm{C}$, which in most cases resulted in exsolution of residual water to form a ring of ice within the sample vial at the top of the solvent. When this occurred, the solvent phase was transferred to a new vial, concentrated further, and then stored at $-20^{\circ} \mathrm{C}$ until analysis. During concentration of these and all other extracts, care was taken to prevent the solvent from evaporating to dryness to limit the loss of semi-volatile organic compounds. Prior to analysis, the concentrated extracts were treated with $\mathrm{Cu}$ powder to remove native sulfur. The $\mathrm{Cu}$ for this treatment was activated by soaking in concentrated $\mathrm{HCl}$ and then rinsed with organic solvents.

After removal of the hydrothermal fluid, the interior of the IGT sampler was rinsed with successive aliquots of methanol $(\mathrm{MeOH})$ and DCM to collect organic compounds that may have precipitated on the walls of the sampler. Both rinses were combined into a single glass vial. In practice, a small amount of residual hydrothermal fluid remained in the sampler that was collected along with the solvents. The combined solvents were evaporated to approximately half their original volume with a stream of $\mathrm{N}_{2}$, at which point the extract usually separated into an $\mathrm{H}_{2} \mathrm{O} / \mathrm{MeOH}$-rich phase underlain by a DCM-rich phase. Additional DCM and acidified Fisher $\mathrm{H}_{2} \mathrm{O}$ were added to the vial to enhance transfer of organic compounds to the DCM phase. To reduce the possibility of contaminating the samples with organic compounds, the $\mathrm{H}_{2} \mathrm{O}$ used for this step was extracted with DCM before adding it to the solvent extract. After shaking, the DCM-rich layer was pipetted into a separate vial and processed using the same methods described for the fluid extract. 
To extract organic compounds precipitated on the walls of the Ti tubing of the POP gun, remaining fluid was removed by suction with a syringe and the tubing was then rinsed with $\sim 5$ $\mathrm{ml}$ aliquots of $\mathrm{MeOH}$ and DCM in succession. The solvents were pushed through the tubing using a solvent-cleaned glass syringe with Teflon plunger. The $\mathrm{MeOH}$ used for this procedure was mildly acidified with $\mathrm{HCl}$ to ensure extraction of carboxylic acids and other weakly acidic compounds present as anions. In some cases, this had the unforeseen consequence of converting organic acids to methyl esters. While most of the $\mathrm{MeOH}$ extracts from the POP gun samples were found to contain small amounts of organic compounds, very few of the DCM extracts contained detectable organic compounds, indicating that most soluble organic compounds were removed from the tubing by the $\mathrm{MeOH}$ rinse.

Because the $\mathrm{MeOH}$ extracts from the POP gun inevitably contained a small amount of hydrothermal fluid that remained in the tubing, the extracts were partially concentrated under a stream of $\mathrm{N}_{2}$, and DCM added. The combined MeOH-DCM extract was then placed in a $-20{ }^{\circ} \mathrm{C}$ freezer to induce phase separation, and the DCM-rich (lower) phase was removed to a clean vial for further processing (note that although DCM was added to these samples, they are still referred to as "MeOH extracts" to reflect the solvent used in the initial extraction step). These extracts as well as the original DCM rinses from the POP gun samples were further concentrated using the same methods described for the IGT fluid samples.

To process the SPE cartridges, hydrothermal fluids remaining in the cartridges was first removed by suction using a large, gas-tight syringe. A glass/Teflon syringe was then used to pass $\sim 10 \mathrm{ml}$ aliquots of $\mathrm{MeOH}$ and DCM through the cartridges in succession. To ensure extraction of organic acid anions, the solvents for this step were mildly acidified with $\mathrm{HCl}$. The $\mathrm{MeOH}$ and DCM rinses were collected in separate pre-cleaned glass vials and processed separately using the methods described above for the IGT and POP gun extracts.

In addition to the fluid samples, organic compounds were extracted from a portion of carbonate chimney from Lost City. The extracted chimney sample was a large fragment (36 g, wet weight) of a small, active flange collected from the Beehive/EXOMAR-12 site adjacent to where the vent fluid samples were collected. Because the flange crumbled into pieces during collection by the ROV, it is not possible to precisely determine what part of the structure the fragment represents, but it was mostly composed of material from the flange interior. The chimney fragment was crushed in a slurry of $80 \% \mathrm{DCM} / 20 \% \mathrm{MeOH}$, transferred to a precleaned glass bottle, and sonicated twice for 10 minutes with vigorous shaking of the sample in between. Following removal of the extract to a separate bottle, the chimney was extracted two additional times using $80 \% \mathrm{DCM} / 20 \% \mathrm{MeOH}$ followed by $100 \% \mathrm{DCM}$. When all of the extracts were combined, the solvents separated into two phases, presumably because of the presence of seawater or vent fluid in the chimney sample. At this stage, the solvent was acidified with $\mathrm{HCl}$ to ensure that alkanoic acids would enter the organic solvent, and the lower, DCM-rich phase was then removed to a separate bottle. In order to remove inorganic salts, the solvent extract was rinsed twice with Fisher DI water that had been pre-extracted with DCM to remove organic compounds. The resulting solvent extract was concentrated under a stream of $\mathrm{N}_{2}$ and prepared for analysis using procedures similar to those used for the fluid samples.

\subsection{Analytical methods}

The solvent extracts were analyzed onshore using gas chromatography-mass spectroscopy (GC-MS) on an Agilent 6890 interfaced to a 5973 mass selective detector. Routine analyses were performed with an Alltech AT-5ms column (60 m length, $320 \mu \mathrm{m}$ internal diameter, 0.25 $\mu \mathrm{m}$ film thickness) using a temperature program of $50{ }^{\circ} \mathrm{C}$ initial temperature for $5 \mathrm{~min}, 10$ 
${ }^{\circ} \mathrm{C} / \mathrm{min}$ to $325{ }^{\circ} \mathrm{C}$, and hold at this temperature for $30 \mathrm{~min}$. For chimney samples, organic acids in an aliquot of the solvent extract were converted to fatty acid methyl esters (FAME) prior to analysis by reaction with $\mathrm{BF}_{3}$ in methanol using kits from Supelco. Compounds were routinely identified by comparison of mass fragment spectra with those in the NIST02 reference library. In a few cases where the spectral identifications were ambiguous (e.g., phenanthrenes, hexadecanol), retention times and mass spectra were confirmed by comparison with analyses of known compounds.

During analysis of extracts by GC-MS, a number of compounds were observed at short retention times $(<24 \mathrm{~min})$ that could be attributed to trace contaminants from the solvents which had been concentrated in the extracts during sample preparation (note that these relatively volatile compounds were lost in some samples that were inadvertently evaporated to dryness). Prominent among these were undecane and several methyldecane isomers that was found to be a trace contaminant in the $\mathrm{MeOH}$ solvent, and several small methylcycloalkanes present in the DCM. Owing to interference from these compounds, low molecular weight organic compounds that may have been present in the hydrothermal fluid samples with short retention times could not be confidently identified during this study. In addition, the sorbents used in the SPE cartridges were found to release relatively large amounts of a spectrum of organic compounds during sample processing, most of which did not match any compounds in the reference library and could not be identified. These contaminants are discussed more fully below, and example mass fragmentograms for the unknown compounds are provided as Supplemental Figure S1.

Compound specific carbon isotope analyses were performed on select samples with an Agilent 6890 coupled to a Delta plus XL mass spectrometer via a combustion interface operated at $1080^{\circ} \mathrm{C}$ using a column and temperature program similar to the GC-MS analyses. Instrument error was estimated using an external standard containing $15 n$-alkanes with known $\delta^{13} \mathrm{C}$ values and was measured to be $0.2 \%$ during the period when these samples were analyzed. All extracts were derivatized using BSTFA $+1 \%$ TMCS and pyridine and heated at $70{ }^{\circ} \mathrm{C}$ for one hour. Results were corrected for the addition of methyl carbon by derivatizing phthalic acid with a known isotopic value using the same BSTFA and subtracting the contribution of methyl carbons by mass balance.

Quantification of fatty acids in selected samples was performed by conversion of the acids to methyl esters and comparison of peak areas for reconstructed ion chromatograms with reference standards for octanoic and dodecanoic acids. Analysis of hydrothermal fluid samples for the presence of dissolved free amino acids was performed by gas chromatography with flame ionization detection following extraction and derivatization of the amino acids with EZ:faast kits from Phenomenex Inc. (Torrance, CA), using the column and analytical parameters specified by the manufacturer. Although an effort was made to detect highly polar compounds by direct injection of small aliquots (1-2 $\mu$ l) of hydrothermal fluid into the GC-MS using an AquaWax column (Alltech), no additional organic compounds were identified by this method.

\subsection{Assessment of potential sources of background contaminants}

In an effort to evaluate potential exogenous sources of organic compounds that might contaminate the hydrothermal fluid samples, samples of bottom seawater were extracted and analyzed using the same procedures utilized for the hydrothermal samples (Fig. 4). A sample of bottom seawater was obtained with the IGT sampler in the vicinity of the TAG field (J2-364IGT6), but no organic compounds were found in either the DCM extract of the fluid or in the solvent rinse of the sampler. Similarly, no organic compounds were observed in a DCM extract of the Fisher $\mathrm{H}_{2} \mathrm{O}$ used in sample processing. Samples of bottom seawater were obtained with 
the POP gun in the vicinity of the Lucky Strike and Lost City vent fields (J2-356-POP1, J2-362POP1). The $\mathrm{MeOH}$ extracts of both seawater samples exhibited a large, unresolved complex mixture (UCM) of organic compounds that were dominated by aliphatic components (Fig. 4a; see Supplemental Fig. S2 for an example mass fragmentogram of the UCM). The UCM displayed three distinct peaks centered at retention times of $\sim 26, \sim 29.5$ and $~ 36$ min. Analyses of the oils used for pressure compensation and hydraulic parts on the Jason submersible are also characterized by broad UCM humps (Figs. 4b and 4c). However, the UCM humps for the oils show very different distribution patterns than those observed for the seawater samples, indicating that the UCM observed in the seawater samples included little or no contribution from the submersible. No organic compounds were found in the DCM extracts of the seawater POP gun samples.

Solvent extracts of the SPE cartridges that were attached to the POP gun during sampling of bottom seawater [(J2-356-SPE(HLB), J2-362-SPE(C8)] contained numerous organic compounds, most of which did not match any compounds in the spectral library used in the GCMS analysis (Figs. 4d and 4e). Similar suites of compounds were observed in all other samples processed through the SPE cartridges, including the hydrothermal fluids and $1 \mathrm{~L}$ samples of Fisher $\mathrm{H}_{2} \mathrm{O}$ pumped through the cartridges and processed using the same procedures. Consequently, the compounds seen in the extracts of the seawater samples appear to be derived predominantly from the HLB and C8 SPE phases rather than the fluid samples, and likely represent fragments of the polymers used to coat the extraction beads. In the case of the C8 phase, the suite of compounds seen in the extracts for all samples included small amounts of $\mathrm{C}_{16}$ and $\mathrm{C}_{18}$ alkenes and alkenones, which also appeared to be derived from the SPE beads. While trace amounts of organic compounds derived from seawater may be present in the SPE samples, they could not be distinguished from the pervasive background of compounds from the SPE phases.

\section{RESULTS}

A summary of the organic compounds that were positively identified in the hydrothermal vent samples is provided in Table 2, with more detailed discussion in the following sections. Note that some compounds in this table were identified using methods that were not applied at all sites, so results for different sites may not be directly comparable. For brevity, only the most salient results of the GC-MS analyses are shown in the figures displayed here; figures showing additional analyses of samples are provided as Supplemental Materials.

The concentration of $\mathrm{Mg}$ in fluid samples collected with the IGT samplers are listed in Table 1. Magnesium concentrations of fluids sampled from deep-sea hydrothermal vents are routinely used to estimate the amount of seawater entrained into the fluid during sampling, based on the presumption that the vent fluids have $\mathrm{Mg}$ concentration approaching zero as a result of fluid-rock interactions in the subsurface while seawater has a concentration of $\sim 52.2 \mathrm{mmol} / \mathrm{kg}$ (German and Seyfried, 2014). However, analysis of IGT samples obtained for this study indicates that endmember vent fluids at the Rainbow field may have $\mathrm{Mg}$ concentrations of $1.5-2 \mathrm{mmol} / \mathrm{kg}$ (see Seyfried et al., 2011). Only two of the samples collected with the IGT samplers had elevated Mg contents (US4, Kremlin) indicating a substantial seawater component. The remaining samples have $\mathrm{Mg}$ concentrations $<8 \mathrm{mmol} / \mathrm{kg}$ and most have concentrations of $2.6 \mathrm{mmol} / \mathrm{kg}$ or less, indicating nearly pure hydrothermal fluids were obtained. Also listed in Table 1 for reference are measured $\mathrm{pH}$ values (at $25{ }^{\circ} \mathrm{C}$ ) and endmember concentrations of dissolved $\mathrm{H}_{2}, \mathrm{CO}_{2}$, and 
$\mathrm{CH}_{4}$ in the fluids determined from separate IGT samples obtained at the same chimney orifices as the organic samples (Reeves et al., 2014).

\subsection{Lost City}

Lost City was the only vent field where significant amounts of extractable organic compounds were consistently observed in the hydrothermal fluid samples. The initial liquidliquid extracts of hydrothermal fluids from the Beehive site at Lost City collected with the IGT samplers yielded no detectable organic compounds (Fig. 5a), but several compounds were present in the sample from the top of Poseidon, including trithiolane, hexadecanol, and squalene (Fig. 5e). Furthermore, hexadecanol, squalene, and cholesterol were observed in the solvent rinse of the IGT sampler used to collect fluids at the top of Poseidon (Fig. 5f), indicating that a fraction of these compounds may have precipitated onto the walls of the sampler. No organic compounds were observed in solvent rinses of the IGT samplers for the Beehive site.

Following acidifcation and re-extraction of the Beehive and Poseidon fluids, a suite of three alkanoic acids was observed that included $n$-octanoic, $n$-decanoic and $n$-dodecanoic acids $\left(\mathrm{C}_{8}\right.$, $\mathrm{C}_{10}$, and $\mathrm{C}_{12}$, respectively) (Fig. $5 \mathrm{~b} \& 5 \mathrm{f}$ ). These compounds were particularly abundant in the samples from the Beehive site (Fig. 5b), with relatively low levels observed in the sample from the top of Poseidon (Fig. 5f). Both fluid samples from Beehive yielded similar alkanoic acid concentrations that decreased with increasing carbon number $\left(\mathrm{C}_{8}>\mathrm{C}_{10}>\mathrm{C}_{12}\right)$ from values of 0.72 to $0.23 \mu \mathrm{mol} / \mathrm{L}$ (Table 3 ). In both samples, acid concentrations decreased with increasing carbon number $\left(\mathrm{C}_{8}>\mathrm{C}_{10}>\mathrm{C}_{12}\right)$. Since it is not clear that the extraction methods completely removed the acids from these fluid samples, the reported concentrations should probably be regarded as minimum values. The alkanoic acids are characterized by relatively heavy carbon isotope compositions, with values between $-2.8 \%$ and $-7.6 \%$ (Table 3 ). The extract of the acidified fluid for the Poseidon sample also included trithiolane, hexadecanol, and squalene as well as trace amounts of cholesterol, indicating that the initial liquid-liquid extraction prior to acidification did not completely remove these compounds (Fig. 5f).

Conspicuously absent from the Lost City IGT sample extracts were alkanoic acids with fewer than 8 or greater than 12 carbon atoms (e.g., $\mathrm{C}_{6}, \mathrm{C}_{14}$ ). To evaluate whether the absence of these compounds may have been an artifact of the analytical protocols, an artificial seawater solution with $\mathrm{pH}$ adjusted to 9.5 containing $\sim 200 \mu \mathrm{g} / \mathrm{L}$ each of $\mathrm{C}_{6}, \mathrm{C}_{12}$, and $\mathrm{C}_{14}$ alkanoic acids was prepared and processed with the same methods used for the natural samples. Quantitative analyses of the resulting extracts showed that recovery of the $\mathrm{C}_{14}$ acid was essentially identical to the $\mathrm{C}_{12}$ acid, implying that the absence of the $\mathrm{C}_{14}$ acid in the Lost City extracts accurately reflects its abundance in these samples. On the other hand, recovery of the $\mathrm{C}_{6}$ acid was only about $40 \%$ relative to the $\mathrm{C}_{12}$ acid, perhaps because extraction of the $\mathrm{C}_{6}$ acid from the water phase by the solvent was less efficient owing to its relatively higher polarity or because this compound was partially volatilized during sample concentration. Nevertheless, even partial recovery of the $\mathrm{C}_{6}$ acid suggests that it would have been detected in the samples if it had been present at levels comparable to the other acids. Accordingly, it appears that levels of both the $\mathrm{C}_{6}$ and $\mathrm{C}_{14}$ acids in the Lost City hydrothermal fluids were significantly lower than those of the $\mathrm{C}_{8}-\mathrm{C}_{12}$ acids.

The two POP gun samples at Lost City (J2-361-POP1, J2-361-POP2) were both obtained at the Beehive site, and the $\mathrm{MeOH}$ extracts were found to contain $\mathrm{C}_{10}$ and $\mathrm{C}_{12}$ alkanoic acids similar to those observed in the IGT extracts from this location (Fig. 5c). However, the relative proportions of these compounds were reversed from the IGT samples, with the $\mathrm{C}_{10}$ acid present in much lower amounts than $\mathrm{C}_{12}$. The $\mathrm{C}_{8}$ alkanoic acid was not observed in either $\mathrm{MeOH}$ extract 
of the POP gun samples. No organic compounds were detected in DCM extracts of the POP guns.

Despite the presence of contaminants from the extraction phase, organic compounds that could be confidently attributed to the hydrothermal fluid were identified in the Beehive sample that was pumped through the HLB SPE phase [J2-361-SPE(HLB)] and extracted with MeOH (Fig. 5d). This sample contained large amounts of the same suite of $\mathrm{C}_{8}, \mathrm{C}_{10}$, and $\mathrm{C}_{12} n$-alkanoic acids that were seen in the IGT samples, and these compounds were present at levels far above those of any background contaminants. Estimates of the concentrations of the acids in the hydrothermal fluid were calculated from the measured abundance of the compounds in the extract and the amount of fluid pumped through the SPE cartridge $(\sim 1.2 \mathrm{~L})$. The resulting concentrations are similar to those measured for the Beehive IGT samples, and show the same decrease in concentration with increasing carbon number (Table 3). No organic compounds were identified in the DCM extract of the HLB phase other than those that could be attributed to background sources. Fluids from the Beehive site were also pumped through a cartridge containing the C8 SPE microbeads [J2-361-SPE(C8)]. However, no identifiable compounds that could be attributed to the hydrothermal fluid were found in solvent extracts of this phase (see Supplemental Fig. S3).

To aid in evaluation of potential sources for alkanoic acids observed in the Lost City vent fluids, organic compounds were extracted and analyzed from a piece of carbonate chimney collected from the Beehive site where the IGT and POP gun samples were obtained. As shown in Figure 6a, analysis of the total extract by GC-MS was dominated by a UCM, although several individual hydrocarbons could be identified including $n$-hexadecane and squalene. Also prominent in the total extract are a number of wax esters (Fig. 6a). These compounds apparently formed through condensation of alkanoic acids with alkanols and alkenols in the extracts induced by acidification of the solvents.

In order to determine the distribution and diversity of alkanoic acids in the chimney, the acids in an aliquot of the total extract were converted to fatty acid methyl esters (FAME) (Figs. 6b and 6c). The FAME analysis revealed an assortment of alkanoic and alkenoic acids ranging in carbon number from $\mathrm{C}_{8}$ to $\mathrm{C}_{28}$, with a strong predominance for compounds with an even number of carbon atoms. The $\mathrm{C}_{14}, \mathrm{C}_{16}$, and $\mathrm{C}_{18}$ compounds are substantially more abundant than compounds with other numbers of carbon atoms, and occur in approximately equal proportions. There is a sharp drop in abundance of the alkanoic acids with fewer than 14 carbon atoms. While it is possible that this drop off could be partially explained by loss of the relatively volatile shorter-chain acids during concentration of the sample, the same procedures used to minimize loss of semi-volatile compounds in the hydrothermal fluid extracts were employed in sample preparation of the chimney extract, suggesting that the relative amounts of these compounds are likely to reflect their in situ abundance. The $\mathrm{C}_{14}-\mathrm{C}_{18}$ carboxylic acids in the chimney extract had carbon isotopic compositions between $-13.5 \%$ and $-20 \%$, substantially lighter than the acids in the fluid (see Supplemental Table S1 for a complete listing of isotopic analyses for the chimney extract).

\subsection{Lucky Strike and Rainbow}

Most solvent extracts of the fluids collected at Lucky Strike and Rainbow using the IGT samplers were found to contain either no detectable organic compounds or trace amounts of compounds that appeared to be mostly derived from sources other than the hydrothermal fluid (Fig. 7). Several of the IGT extracts contained small peaks whose mass spectra did not provide a 
close match to any compounds in the spectral library, and were inferred to be contaminants, possibly fragments of plastic polymers (see Supplemental Fig. S1 for an example). The source of these compounds could not be determined. Several of the fluid extracts were also found to contain small amounts of Fluorolube lubricant, but these compounds could be readily identified as contaminants by their mass fragmentation patterns during GC-MS analysis. An example is provided in Figure 7b, which shows the extract for a fluid sample from the Lucky Strike hydrothermal field (J2-359-IGT6). The only identifiable compound in this sample that could not be attributed to Fluorolube or other contaminants was a small peak for dibenzothiophene. Dibenzothiophene was present in one other sample, also from Lucky Strike (J2-358-IGT6), but was not found in any other samples analyzed for this study. Analyses of the solvent rinses of the IGT samplers following removal of the fluid did not reveal the presence of any compounds that could be confidently attributed to a hydrothermal fluid source. Most of these rinse samples, however, did include small amounts of phthalates from an unidentified source as well as traces of Fluorolube.

In contrast to the scarcity of organic compounds in the IGT samples, organic compounds were observed in several of the POP gun samples from sites at Rainbow and Lucky Strike. Extracts from all sites at Rainbow sampled with the POP gun contained small amounts of PAH that included phenanthrene, methylphenanthrenes, and $\mathrm{C}_{2}$-phenanthrenes (e.g., Figs. 8a-c). In addition, samples from the Guillaume vent contained hexadecanol and a $\mathrm{C}_{18}$ alkenoic acid (Fig. 8c). Because the solvents used in the extracts were mildly acidified, the organic acids in this and other samples were converted to methyl esters during sample processing. While it is possible that these compounds represent membrane components of microorganisms entrained into the POP gun during sampling, essentially identical results were obtained from both POP gun samples at this site, indicating it is more likely that the compounds were indigenous to the hydrothermal fluid.

Extracts of the POP gun samples from the high temperature Stylo 2 and Padraig sites at Rainbow also contained a small, narrow UCM hump centered at a retention time of $\sim 36 \mathrm{~min}$ (Fig. 8b). A UCM hump was also evident in both samples from the lower temperature $\left(191^{\circ} \mathrm{C}\right)$ Ecurie site at Rainbow, but in this case the UCM was much broader and extended from 26 to 38 min retention time (Fig. 8a). In all cases, the UCM was dominated by aliphatic components as indicated by a predominance of mass fragments with mass/charge ratios (m/z) of 55, 57, 69, 71, 83, and 85 (see Supplemental Fig. S2). The single POP gun sample obtained at Lucky Strike did not contain a prominent UCM, but did include several even-carbon-numbered alkenoic and alkanoic acids ranging in carbon number from $\mathrm{C}_{14}$ to $\mathrm{C}_{22}$, with $\mathrm{C}_{16}$ and $\mathrm{C}_{18}$ alkenoic acids particularly abundant (Fig. 8d). Since these compounds are predominant components of bacterial membranes, it appears likely that in this case the alkenoic and alkanoic acids must have been extracted from biomass entrained into the POP gun during sampling rather than being dissolved in the hydrothermal fluid.

Among the DCM extracts of the POP gun samples, organic compounds were observed in only some of the Rainbow samples and were not found in any samples from Lucky Strike. Extracts of samples from the high temperature Guillaume and Padraig sites at Rainbow all contained phenanthrene and methylfluorenes present in trace amounts that were just barely detectable (Fig. 9a). However, these compounds were not observed at the other high temperature site sampled, Stylo 1. One of the DCM extracts for the POP gun samples from the lower temperature Ecurie site at Rainbow contained a homologous series of $n$-alkanes ranging in carbon number from 23 to 33 (Fig. 9b). The alkanes exhibited a bell-shaped distribution in 
abundance centered around $\sim \mathrm{C}_{28}$, with no apparent even- or odd-carbon-number preference. These hydrocarbons were not observed in any other POP gun extract. systems contained organic compounds that could be differentiated from the background of compounds derived from the SPE sorbent (see Supplemental Fig. S4). The same suite of background peaks observed in the $\mathrm{MeOH}$ extracts were also evident in the DCM extracts of the HLB phase (Supplemental Fig. S4). In addition, the DCM extracts also included $\mathrm{C}_{16}$ and $\mathrm{C}_{18}$ alkenes that were not present in the $\mathrm{MeOH}$ extracts, but which also appeared to be derived from the extraction phase since they were present in all samples processed through the SPE cartridges.

Despite the ubiquitous presence of background contaminants, organic compounds that could be attributed to the hydrothermal fluids were identified in some DCM extracts of the HLB phase. The DCM extract of sample J2-359-SPE(HLB) from the Medea site at Lucky Strike (Fig. 10b) was found to contain methyl esters of $\mathrm{C}_{16}$ and $\mathrm{C}_{18}$ alkanoic and alkenoic acids similar to those observed in the MeOH extract of the POP gun at this same sample location (Fig. 8d). The DCM extract of the HLB phase obtained from the Ecurie site at Rainbow (Fig. 10a) contained a homologous series of $\mathrm{C}_{23}-\mathrm{C}_{33} n$-alkanes with a distribution identical to that observed in the DCM extract of the POP gun sample for this site (Fig. 9b). In both cases, the compounds identified were not observed in DCM extracts of the SPE cartridges from other sites.

The only high-temperature sample processed through a SPE cartridge containing the C8 sorbent was from the Stylo 2 site at Rainbow [J2-355-SPE(C8)]. Extracts of this sample contained the same suite of compounds observed in all other C8 extracts that appeared to be attributable to background from the sorbent phase (Supplemental Fig. S3). Although there is a broad "window" during the GC-MS analysis between 16 and 30 min retention time where there is little or no background, no organic compounds indigenous to the sample were observed in this interval.

\subsection{Amino acid analyses}

Preserved fluid samples from the IGT samples from all sites were analyzed for the presence of free amino acids by GC-MS following extraction from the fluid and derivatization. However, no free amino acids were detected in any of the hydrothermal fluids or in bottom seawater samples at a detection limit of $5 \mathrm{nmol} \mathrm{kg}{ }^{-1}$. This result is consistent with the absence of detectable free amino acids at similar levels in $319^{\circ} \mathrm{C}$ hydrothermal vent fluids from the Guaymas Basin (Haberstroh and Karl, 1989) and $>200{ }^{\circ} \mathrm{C}$ hydrothermal vent fluids from the Marianas Trough (Fuchida et al., 2014). Conversely, Klevenz et al. (2010) reported several dissolved amino acids present above the $5 \mathrm{nmol} \mathrm{kg}^{-1}$ threshold from high-temperature hydrothermal sites further south on the Mid-Atlantic Ridge, with total free amino acid concentrations up to $377 \mathrm{nmol} \mathrm{kg}^{-1}$. Lang et al. (2013) reported total hydrolysable amino acids (THAA) for hydrothermal fluids from the Lost City system ranging from 736 to $2300 \mathrm{nmol} \mathrm{kg}^{-1}$, but did not report free amino acid concentrations. The apparent absence of detectable free amino acids in the Lost City fluids suggests that the amino acids reported by Lang et al. (2013) are predominantly present as peptides or proteins.

\section{DISCUSSION}

\subsection{Organic compounds in Lost City hydrothermal fluids}

Lost City was the only sampling location in this study where significant amounts of dissolved organic compounds were consistently observed in the hydrothermal fluids. At that site, 
substantial concentrations of $\mathrm{C}_{8}-\mathrm{C}_{12}$ alkanoic acids as well as several other organic compounds (squalene, hexadecanol, trithiolane, and cholesterol) were observed in multiple samples and, in the case of the alkanoic acids, were identified in all samples regardless of the collection and processing methods used (Fig. 5). Although several of the compounds found in the Lost City fluids were also present at low abundance in extracts of the carbonate chimney recovered from that site, the fluid extracts lacked numerous other compounds that were abundantly present in the chimney extract. We therefore conclude that the organic compounds observed in the Lost City samples were indigenous to the hydrothermal fluid and not derived from chimney particles entrained during sampling.

Measured concentrations of the $\mathrm{C}_{8}-\mathrm{C}_{12}$ acids for the two IGT samples and the SPE sample at the Beehive are in good agreement, and indicate concentrations for individual acids in the 0.23 to $0.77 \mu \mathrm{mol} / \mathrm{L}$ range, which is equivalent to 2.7-6.2 $\mu \mathrm{mol} \mathrm{C} / \mathrm{L}$ (Table 3). For comparison, concentrations of dissolved formate and acetate for fluids venting at the Beehive site in 2005 were found to be $\sim 144 \mu \mathrm{mol} / \mathrm{L}$ and $8.5 \mu \mathrm{mol} / \mathrm{L}$, respectively (Lang et al., 2010). The measured concentration of dissolved organic carbon (DOC) in the fluids sampled in 2005 was $\sim 102 \mu \mathrm{mol}$ $\mathrm{C} / \mathrm{L}$, of which $\sim 50 \mu \mathrm{mol} \mathrm{C} / \mathrm{L}$ can be accounted for as formate and acetate (note that some of the formate and acetate are lost during sample preparation for DOC analysis) (Lang et al., 2010). Collectively, the $\mathrm{C}_{8}-\mathrm{C}_{12}$ acids can account for $\sim 11-15 \mu \mathrm{mol} \mathrm{C} / \mathrm{L}$, or $11-15 \%$ of the DOC (Table 3). Taken together, it appears that the carboxylic acids that have been measured so far (including formate) can account for $>60 \%$ of the DOC in the hydrothermal fluids at the Beehive site. Total hydrolysable amino acids can account for an additional 3-5\% of DOC (Lang et al., 2013), leaving about one third of the total DOC still unaccounted for at this point. A number of alkylated monocyclic compounds have been identified in the Lost City fluids using other methods (Konn et al., 2009) that might contribute to the remaining third, or it could be composed of highly polar molecules (e.g., pyruvate, alkanols) not detectable by the methods employed to date. Alternatively, the sampling methods used may have underestimated the amounts of carboxylic acids present.

The detection of only even-carbon-numbered alkanoic acids in the Lost City samples indicates that the acids have a biological source. Although abiotic organic synthesis reactions such as the Fischer-Tropsch process are known to produce long-chain carboxylic acids (e.g., McCollom et al., 1999, 2010), these reactions typically produce an mixture of even- and oddcarbon-numbered compounds in roughly equal amounts as the result of sequential addition of single carbon units (McCollom and Seewald, 2007). There is currently no known mechanism for abiotic synthesis of organic compounds in two-carbon units that might produce exclusively evencarbon-numbered compounds, nor is there any evidence from natural organic products thought to have an abiotic abiotic origin for an even-number predominance. For instance, abiotic organic compounds in meteorites have approximately equal proportions of even- and odd-carbonnumbers (Yuen and Kvenvolden, 1973; Huang et al., 2005). Furthermore, the absence of other types of even-carbon-numbered compounds (e.g., hydrocarbons) makes it unlikely that the acids come from an abiotic source.

Since the $\mathrm{C}_{8}-\mathrm{C}_{12}$ alkanoic acids are too small to be components of lipid membranes and are not known as common metabolic products of microbial metabolism, the biological source for the compounds is unclear. However, these compounds are known to be inhibitors of some metabolic pathways such as fermentation (e.g. Legras et al., 2010), and perhaps they play a similar role in the Lost City microbial community. These compounds are probably produced by bacteria, since archaea do not appear to synthesize linear alkanoic acids. Studies of the microbial communities 
at the hottest Lost City vents $\left(70-91^{\circ} \mathrm{C}\right)$ have found that the interiors of the carbonate chimneys are dominated by a single species of archaea related to the Methanosarcinales, but the chimney exteriors and hydrothermal fluids contain bacteria that could be the source of the alkanoic acids (Schrenk et al., 2004; Brazelton et al., 2006). Alternatively, the compounds might be produced in the subsurface. In either case, the presence of $\mathrm{C}_{8}-\mathrm{C}_{12}$ alkanoic acids at relatively high abundance may reflect novel metabolic pathways taking place in this high $\mathrm{pH}$ ecosystem. Another possibility is that the compounds are formed through thermal decomposition of bioorganic matter in the subsurface. In this respect, it may be notable that recent studies of insoluble carbonaceous matter from subseafloor serpentinites have found that they contain a large component of aliphatic compounds up to $\mathrm{C}_{12}$ associated with carboxylate functional groups (Pasini et al., 2013).

The alkanoic acids in the fluid and chimney samples from Lost City form complementary sets, with the fluid containing $\mathrm{C}_{8}, \mathrm{C}_{10}$, and $\mathrm{C}_{12}$ acids but none of the longer chain acids, while the chimney contained substantial amounts of $\mathrm{C}_{14}, \mathrm{C}_{16}$, and $\mathrm{C}_{18}$ acids with only small amounts of the shorter chain acids (Figs. 5 and 6). This result suggests two possible origins for the acids dissolved in the Lost City hydrothermal fluids. The first possibility is that a complete suite of $\mathrm{C}_{8}$ to $\mathrm{C}_{18}$ acids is produced by biological processes occurring within the chimney walls, but the shorter acids are preferentially dissolved by the circulating hydrothermal fluids owing to their relatively higher aqueous solubility. Alternatively, all of the acids may be produced by biological activity deeper in the system that are mobilized by the hydrothermal fluids, but only the longer-chain acids are subsequently removed by precipitation as the fluids cool during mixing with seawater within the walls of the chimney (a mechanism that would be directly comparable to that used as the basis for the design of our POP gun sampling device). In the former case, mobilization of the shorter acids would represent a significant loss of fixed organic carbon from the microbial communities living in the chimney walls, while the latter case would represent a significant net transfer of organic carbon from deep within the system to the seafloor chimney environment.

The carbon isotopic compositions of the $\mathrm{C}_{8}-\mathrm{C}_{12}$ alkanoic acids do not allow for clear discrimination between these two possibilities. The $\mathrm{C}_{8}-\mathrm{C}_{12}$ acids in the fluid are relatively heavy compared to the $\mathrm{C}_{14}-\mathrm{C}_{18}$ acids in the adjacent chimney ( $-2.8 \%$ o to $-7.6 \%$ vs. $-13.5 \%$ o to $-20 \%$ ) (Fig. 11). However, the $\mathrm{C}_{16}$ and $\mathrm{C}_{18}$ acids in other chimney samples from Lost City analyzed by Bradley et al. (2009) overlap with the heavier values observed in the fluids, with some values as low as $-1.1 \%$ (Fig. 11). Thus, it appears possible, and maybe even likely, that the $\mathrm{C}_{14}-\mathrm{C}_{18}$ acids in the Lost City chimneys are composed of a mixture of heavier compounds from the same source as the acids observed in the hydrothermal fluid and lighter compounds derived from in situ microbial activity, with the relative contributions from these sources varying locally. Within this scenario, however, the ultimate source of the heavier acids could either be local to the chimney deposits or somewhere deeper within the system.

The IGT fluid sample collected at the top of the Poseidon structure contained substantially lower amounts of the $\mathrm{C}_{8}-\mathrm{C}_{12}$ alkanoic acids than the samples collected from the Beehive site on the side of Poseidon (Fig. 5), and also contained a number of compounds not observed in the other samples including squalene, trithiolane, hexadecanol, and cholesterol. The fluid from the Poseidon summit also had a substantially lower temperature $\left(51^{\circ} \mathrm{C}\right.$ vs. $91^{\circ} \mathrm{C}$ ), indicating the fluid had conductively cooled or mixed with cold seawater during passage through the length of the structure. Squalene is a common product of the microbial community living within Lost City chimney structures (Méhay et al., 2013), and microbial activity within the chimney structure is 
presumably the source of this compound as well as the hexadecanol and cholesterol. Although $\mathrm{O}_{2}$ is required for biosynthesis of cholesterol, influx of seawater into chimney mixing zones could supply $\mathrm{O}_{2}$ for aerobic organisms, and the cholesterol was likely entrained into circulating fluids from these zones. Microbial or other abiotic processes occurring within the chimney may have also led to partial loss of alkanoic acids in the fluid. To our knowledge, trithiolane has not previously been reported to occur in deep-sea hydrothermal systems. However, we have observed trithiolane and several other cyclic carbon-sulfur compounds in strongly alkaline $(\mathrm{pH}>$ 11) fluids discharged from serpentinite-hosted springs at Aqua de Ney in northern California (Feth et al., 1961; Barnes et al., 1972), suggesting these compounds may be common in fluids discharged from serpentinites (Fig. 5g). Whether the trithiolane observed in the Lost City fluid is a biological or abiotic product is presently unclear.

\subsection{Organic compounds in high-temperature hydrothermal fluids}

The high-temperature $\left(\geq 190{ }^{\circ} \mathrm{C}\right)$ hydrothermal fluids examined for this study were characterized by an overall scarcity of extractable dissolved organic compounds. Most samples were found to contain either no detectable organic compounds or only trace amounts of organic compounds composed primarily of polycyclic aromatic hydrocarbons. More substantial amounts of organic compounds were observed in a few of the POP gun and SPE samples but, as discussed further below, these compounds appeared to represent particulate matter or biomass entrained during sample collection rather than dissolved components present in the hydrothermal fluids.

Several of the $\mathrm{MeOH}$ extracts from the POP gun samples at Rainbow were found to contain unresolved complex mixtures of organic compounds (e.g., Figs. 8a and 8b). The UCMs observed for the hydrothermal samples have some similarities to the UCM found in the POP gun samples of deep seawater, but there are also some substantial differences. The UCM observed for bottom seawater displayed a broad hump extending from $\sim 25$ to $\sim 37$ min retention time, with three distinct peaks centered at $\sim 26, \sim 29.5$ and $\sim 36$ min (Fig. 4a). The UCM for the moderately hot fluids $\left(191^{\circ} \mathrm{C}\right.$ ) from the Ecurie site also displayed a broad hump extending from $\sim 25$ to $~ 37$ min (Fig. 8a). However, while the Ecurie samples display a distinct peak at 36 min similar to that for seawater, there is only a slight indication of a peak at 29.5 min and none at $26 \mathrm{~min}$. Conversely, the UCM humps evident in the samples from the $\geq 350{ }^{\circ} \mathrm{C}$ Stylo 2 and Padraig sites are much narrower, with a single peak at 36 min that coincides closely with the final peak in the seawater UCM (Fig. 8b). For both seawater and hydrothermal fluid samples, mass fragmentation patterns from GC-MS analyses show that the UCMs are dominated by saturated alkyl $(\mathrm{m} / \mathrm{z}=57$, 71 , 85, etc.) and monounsaturated alkenyl ( $\mathrm{m} / \mathrm{z}=55,69$, 83, etc.) fragments, indicating the compounds that comprise the UCMs have a large aliphatic component (see Supplemental Fig. S2). This observation appears to be consistent with studies indicating that dissolved organic matter (DOM) in deep Atlantic Ocean water contains a large aliphatic component (Hertkorn et al., 2013), although the actual molecular structures of the compounds making up the UCM remain to be determined.

The similarities between the hydrothermal and seawater UCMs suggest that the mixture of compounds that comprise the hydrothermal UCMs may represent seawater organic matter that has been modified by high-temperature processes or biological activity during circulation though the hydrothermal system. If this is the case, the hydrothermal samples appear to have preferentially lost the lower molecular weight fraction of the UCM, with more extensive loss occurring at the higher temperature Stylo 2 and Padraig sites than at the lower temperature Ecurie site. Regardless, differences between the seawater and hydrothermal UCMs indicate that the organic compounds found in the hydrothermal samples do not simply represent seawater 
organic matter entrained during sample processing or shallow mixing of seawater into the fluids. In contrast, the fluids from Lucky Strike and Lost City contained no apparent UCM, suggesting that the compounds that constitute seawater UCM are quantitatively removed during circulation through these systems.

Although the UCMs observed in the POP gun samples from Ecurie represent the largest amount of organic matter observed in any of the high-temperature fluid samples in this study, these compounds appear to be derived from particulate materials entrained during sampling rather than compounds dissolved in the hydrothermal fluid. Extracts of hydrothermal chimneys from the Rainbow field also contain substantial UCM humps (Simoneit et al., 2004). While direct comparisons are complicated by differences in analytical protocols, the UCM humps for many chimney samples reported by Simoneit et al. (2004) are broad with a peak towards the higher molecular weight fraction, and appear to be very similar to the UCMs observed for the Ecurie samples. In addition, many of the chimney samples display a suite of linear alkanes ranging in carbon number from $\sim \mathrm{C}_{22}$ to $\sim \mathrm{C}_{33}$. This suite of alkanes displays a bell-shaped distribution in abundance centered at about $\mathrm{C}_{28}$, with no even-odd carbon number preference. The carbon number range and abundance distribution of these compounds is nearly identical to the suite of $n$-alkanes observed in the DCM extracts of the POP gun and SPE samples at the Ecurie site (Figs. 9b and 10a). Although long-chain alkanes are usually associated with plant waxes, Simoneit et al. (2004) suggested that their occurrence in the chimney deposits may reflect thermal processing of organic matter within the Rainbow hydrothermal environment. Conversely, a direct microbial source for these longer-chain compounds cannot be precluded.

Combined, the UCMs and alkanes observed in the Ecurie fluids provide a very close match to the organic compounds extracted from chimneys at Rainbow, suggesting a common source for the organic compounds observed in the chimney deposits and the POP gun and SPE samples. While most of the fluids collected for this study were sampled directly from natural orifices, at Ecurie the fluids were sampled from a cavity excavated into the side of a chimney structure that was weakly venting lower temperature fluids $\left(\sim 60^{\circ} \mathrm{C}\right)$ at its apex (Fig. 1b). The tip of the POP gun was embedded in loose chimney materials during sampling, and it is likely that some chimney particles were entrained into the POP gun and SPE cartridge during sampling, and are the source of the UCM and alkanes observed in the POP gun and SPE extracts. If this is the case, it suggests that a substantial fraction of the UCM found in the hydrothermal chimneys could be seawater-derived organic matter that has undergone alteration to remove some of the lower molecular weight fraction. Whether this alteration is primarily chemical or biological remains to be determined.

The source of smaller UCMs observed in POP gun samples from the higher temperature Stylo 2 and Padraig sites at Rainbow is more difficult to evaluate (Fig. 8b). The POP gun extracts for these sites also contain several PAHs, and the narrow UCM peak and lack of alkanes observed in the POP gun samples distinguishes them from the suite of organic compounds reported for chimney samples. These observations suggest that the UCM and PAH found in the extracts were present as dissolved compounds in the fluids.

Extracts of the two POP gun samples from the Guillaume site at Rainbow contained hexadecanol and a $\mathrm{C}_{18}$ alkenoic acid in addition to several PAH (Fig. 8c). Hexadecanol was not observed in other samples from Rainbow, but it was observed in the fluid sampled from the top of Poseidon at Lost City (Fig. 5e-g). While hexadecanol is a plausible microbial product, it is difficult to explain how this compound would occur in isolation without being accompanied by a suite of other alkanols or alkanoic acids if derived from a biological source. On the other hand, 
there is no obvious source of contamination that would explain the occurrence of this compound in the samples. Thus, the hexadecanol appears to be indigenous to the samples, but the ultimate source for this compound remains obscure. Similarly, the $\mathrm{C}_{18}$ alkenoic acid found at the Guillaume site appears to be an indigenous biological product, but its source is uncertain.

Overall, several PAH, particularly phenanthrene together with its methyl and dimethyl homologs, were the only organic compounds consistently found in the high-temperature fluids from the Rainbow field that appeared to be indigenous to the fluids (e.g., Figs. 8 and 9). Although the PAHs were not quantified, they were present at very low levels that were barely detectable in the extracts. Phenanthrene and other PAHs are prominent constituents of organic matter that has been exposed to high-temperature alteration in hydrothermal environments (Simoneit, 1988; Kawka and Simoneit, 1994; Ventura et al., 2012), suggesting that the PAH observed in the fluids at Rainbow may represent organic matter that has undergone thermal processing during circulation through the hydrothermal system. Because this process obscures the original composition of the organic matter, it is difficult to identify the ultimate source of these compounds.

At the Lucky Strike site, the hydrothermal fluid extracts were largely devoid of organic compounds that could be attributed to the fluids with a high degree of confidence, with the possible exception of trace dibenzothiophene that was observed in a couple of samples (e.g., Fig. 7b). The only POP gun sample obtained at Lucky Strike (J2-359-POP1) was found to contain a number of fatty acid methyl esters, dominated by $\mathrm{C}_{16}$ and $\mathrm{C}_{18} n$-alkanoic and $n$-alkenoic acids (Fig. 8d) (note that fatty acids present in the sample were probably converted to methyl esters during sample processing). These compounds were also observed in the SPE sample attached to the POP gun (Fig. 10b), but not in the IGT fluid sample obtained at the same vent site (Fig. 7b). The similarity of the fatty acids found in the POP gun and SPE samples to the components of bacterial membranes strongly suggests that these compounds were derived from microbial biomass entrained during sample collection with the POP gun rather than representing dissolved organic compounds from the hydrothermal fluid.

In addition to an aliphatic component, deep seawater DOM has also been found to contain a significant component of polycyclic aromatic compounds with attached carboxyls and other functional groups (e.g., Dittmar and Koch, 2006). It has been hypothesized that some of these compounds could originate in submarine hydrothermal systems. Our results do not show evidence for a substantial input of aromatic compounds into the deep sea from high temperature hydrothermal systems, since such compounds were only detected in trace amounts. However, more substantial amounts of PAH could be contributed from hydrothermal systems that are buried by organic-rich sediments where fluids may discharge much higher amounts of aromatic compounds (Simoneit, 1988).

\subsection{Comparison with previous studies}

The results of this study stand in sharp contrast with previous studies that have reported the presence of diverse organic compounds in fluids from the Rainbow and Lost City vent fields, as well as for a hydrothermal system on the East Pacific Rise (Brault et al., 1988; Konn et al., 2009, 2012). Konn et al. (2012) extracted small aliquots $(10 \mathrm{ml})$ of hydrothermal fluids from the Rainbow field using a stir-bar solid phase extraction approach, and reported detection of a diverse suite of organic compounds that included alkanes, alkanoic acids, cycloalkanes, and aromatic hydrocarbons. The alkanoic acids observed ranged from $\mathrm{C}_{9}$ to $\mathrm{C}_{18}$, with a predominance of even carbon number and particularly high abundances of $\mathrm{C}_{12}, \mathrm{C}_{14}$, and $\mathrm{C}_{16}$ acids. In contrast, we observed no alkanoic acids in the Rainbow samples, despite extraction of 
much larger sample volumes ( $>10 \times$ larger volumes for the IGT fluid samples, $~ 100 \times$ larger for the POP gun and SPE samples). Although Konn et al. (2012) did not quantify the amount of acids in their samples, the relatively strong GC-MS response they observed for the $\mathrm{C}_{12}-\mathrm{C}_{16}$ acids suggests that these compounds would have been readily detectable in our samples if they had been present at comparable levels (or even at substantially lower levels).

Konn et al. (2012) also reported observation of a homologous series of $n$-alkanes ranging from $\mathrm{C}_{9}$ to $\mathrm{C}_{19}$, with especially high abundances of $\mathrm{C}_{10}, \mathrm{C}_{11}$, and $\mathrm{C}_{12}$ alkanes at Rainbow. We observed $n$-alkanes in only one Rainbow sample (Ecurie), and these compounds had a much higher range of carbon numbers $\left(\mathrm{C}_{23}-\mathrm{C}_{33}\right)$. Alkanes in the carbon number range reported by Konn et al. (2012) were not found in any of the hydrothermal fluids we sampled at Rainbow (note that undecane and several methlydecanes were observed in all of our samples wherever $\mathrm{MeOH}$ was used as a solvent, but these compounds were identified as contaminants from the solvent and were not derived from the fluids). In agreement with our results, Konn et al. (2012) also reported the observation of trace amounts of PAHs, but found a much broader spectrum of compounds than the limited number observed during this study (primarily phenanthrene and alkylated homologs; Table 2). Although monocyclic alkylated aromatic compounds and cycloalkanes were prominent components of the suite of organic compounds reported by Konn et al. (2012), the methods employed for this study were not suited for identification of compounds of that type.

In an earlier study, Konn et al. (2009) had reported detection of a suite of compounds at Lost City that were very similar to those they identified at Rainbow. Again, their results are very different from those found in the present study. While Konn et al. (2009) reported alkanoic acids to be present in the Lost City fluid, these compounds were only present at low levels and were dominated by the $\mathrm{C}_{12}, \mathrm{C}_{14}$, and $\mathrm{C}_{16}$ acids rather than the $\mathrm{C}_{8}, \mathrm{C}_{10}$, and $\mathrm{C}_{12}$ acids found to be dominant in this study. Konn et al. (2009) report carbon isotopic compositions for the $\mathrm{C}_{8}-\mathrm{C}_{12}$ alkanoic acids, but their reported values $\left(\mathrm{C}_{8}=-31.1 \%, \mathrm{C}_{10}=-19.6 \%\right.$, and $\mathrm{C}_{12}=-25.5 \%$ ) are considerably lighter than the compositions measured for the same compounds in this study (Table 3), although the values reported by Konn et al. are similar to the longer-chain alkanoic acids in the Lost City chimney extract. Konn et al. (2009) also report detection of a number of alkanes and PAH at Lost City, but none of those were observed in the samples analyzed for this study.

Some key aspects of the organic compounds identified in the Rainbow and Lost City fluids by Konn et al. (2009, 2012) are particularly noteworthy. First, nearly identical suites of organic compounds were reported for hydrothermal fluids in both systems, even though the fluids have radically different chemistries and have experienced very different hydrothermal circulation histories (see, for example, Fig. 9 of Konn et al., 2009). End-member hydrothermal fluids at Rainbow attain temperatures well in excess of $365^{\circ} \mathrm{C}$ and are among the most acidic and metalrich observed in mid-ocean ridge hydrothermal systems worldwide (Table 1; Charlou et al., 2002). In contrast, the hydrothermal fluids discharged from the Lost City system have apparently experienced maximum temperatures of about $150-250{ }^{\circ} \mathrm{C}$ during circulation through the subsurface and have become strongly alkaline through the process of serpentinization (Kelley et al., 2005; Proskurowski et al., 2006; Foustoukos et al., 2008). There is no obvious reason to expect that the organic composition of the Rainbow and Lost City fluids should be similar, and it would be truly remarkable if fluids experiencing such radically different conditions were characterized by the same organic content. 
Second, the suite of organic compounds reported by Konn et al. has some highly unusual characteristics that would be difficult to explain by natural processes. For instance, the $n$-alkanes are dominated by $\mathrm{C}_{10}$ and $\mathrm{C}_{11}$ compounds in both systems, with much lower amounts of other $n$ alkanes. Typically, natural sources of organic compounds display much broader and more regular distributions of $n$-alkanes, with peaks in distribution at higher carbon number (e.g., Simoneit et al., 2004; Delacour et al., 2008). Conversely, abiotic organic synthesis pathways produce mixtures of compounds that display a regular decrease in alkane abundance with increasing carbon number rather than having a peak at a discrete number of carbons (e.g., McCollom et al., 2010). A natural source for high abundances of $C_{10}$ and $C_{11} n$-alkanes to the exclusion of lower and higher carbon number compounds is difficult to explain. Also, while homologous series of alkanes and alkanoic acids in geologic samples tend to exhibit regular patterns of abundance with carbon number (e.g., Brault et al., 1988; Simoneit et al., 2004), the homologues reported by Konn et al. $(2009,2012)$ exhibit no regular or consistent pattern. For example, the alkanoic acids show no regular abundance with carbon number, and the $\mathrm{C}_{10}, \mathrm{C}_{11}$, and $\mathrm{C}_{12} n$-alkanes appear to be present in roughly equal amounts while those with both fewer $\left(\mathrm{C}_{9}\right)$ and greater $\left(\mathrm{C}_{13}-\mathrm{C}_{19}\right)$ are present in substantially lower amounts (see Fig. 5 in Konn et al., 2012). It is somewhat difficult to conceive of a natural process that could explain the irregular distributions observed for these compounds.

It is not clear how to reconcile the discrepancies between the results of the present study and those reported for the same sites by Konn et al. (2009, 2012). The discrepancies may reflect real temporal or spatial differences in the organic composition of the hydrothermal fluids that were sampled, or differences in the effectiveness of the protocols to extract and detect organic compounds from the fluids. However, the successful detection of organic compounds in the samples from Lost City and other sites indicates that the methods used in the present study were effective in extracting dissolved organic compounds when they are present, suggesting that it is unlikely that our study would have missed the compounds reported by Konn et al. (2009, 2012) if they had been present. Alternatively, the unusual distribution of compounds and close similarities between different sites observed by Konn et al. $(2009,2012)$ may be an indication that many of the compounds they reported are derived from an unrecognized background source of contamination rather than the hydrothermal fluids. Further comparative tests of the extraction and analytical protocols employed in these various studies as well as analysis of additional samples of hydrothermal fluids using multiple methods are needed to resolve this issue.

In an earlier study, Holm and Charlou (2001) reported observation of trace amounts of $\mathrm{C}_{16}$ to $\mathrm{C}_{29} n$-alkanes in samples of high-temperature $\left(364^{\circ} \mathrm{C}\right)$ hydrothermal fluids from the Rainbow field. These compounds were extracted from $50 \mathrm{ml}$ aliquots of hydrothermal fluids that had been stored in a cold room for a considerable length of time prior to analysis. Organic compounds were extracted from the fluid with SPE units containing the same HLB sorbent used in this study. In the present study, alkanes were not identified in any of the high-temperature (326-366 ${ }^{\circ} \mathrm{C}$ ) hydrothermal fluids sampled at Rainbow, even in the sample from Stylo2 where more than a liter of fluid was pumped through the SPE cartridge containing HLB. The only site where alkanes were observed at Rainbow was at the lower temperature Ecurie site (Figs. 9b and 10a), but the alkanes in those samples have a substantially different carbon number distribution $\left(\mathrm{C}_{22}\right.$ to $\mathrm{C}_{33}$ ) from those reported by Holm and Charlou (2001), and are most likely derived from chimney particulates entrained during sampling. Consequently, we are unable to reproduce or confirm the results of Holm and Charlou (2001). 
863

864

865

866

867

868

869

870

871

872

873

874

875

876

877

878

879

880

881

882

883

884

885

886

887

888

889

890

891

892

893

894

895

896

897

898

899

900

901

902

903

904

905

906

907

908

The only other study to provide a detailed analysis of organic matter extracted from deep-sea hydrothermal vent fluids is Brault et al. (1988). Those authors analyzed dissolved organic compounds in $>250{ }^{\circ} \mathrm{C}$ fluid venting from a basalt-hosted hydrothermal system at $13^{\circ} \mathrm{N}$ on the East Pacific Rise collected with a $350 \mathrm{ml}$ Ti syringe sampler and extracted with a liquid-liquid method using chloroform as the solvent. The extract contained a suite of $n$-alkanes ranging in size from $\mathrm{C}_{17}$ to $\mathrm{C}_{35}$ along with an unconsolidated complex mixture (UCM) of unidentified compounds dominated by aliphatic components. The UCM hump in the extract was broad with a peak toward the higher molecular weight end centered at about $\mathrm{C}_{29}$. The alkanes exhibited a smooth distribution with a peak in abundance at about $\mathrm{C}_{28}$, with a very slight preference for even carbon number compounds. The total amount of aliphatic hydrocarbons in their hydrothermal fluid sample was $\sim 56 \mu \mathrm{g} \mathrm{l}^{-1}$, which was 100 times greater than levels in the local seawater.

The suite of organic compounds observed by Brault et al. (1988) were not observed in any of the hydrothermal fluids examined in the present study using comparable liquid-liquid extraction methods. Although Brault et al. (1988) extracted a larger volume of hydrothermal fluid than was included in the IGT samples (350 ml vs. 120 ml) analyzed here, if aliphatic hydrocarbons had been present in comparable amounts to those reported by Brault et al. they should have been readily detectable in the IGT extracts. Samples from the Ecurie site at Rainbow were the only samples in this study where a suite of compounds similar to those reported by Brault et al. (1988) were identified. These samples contained both a suite of alkanes peaking at $\sim \mathrm{C}_{28}$ and a broad UCM with a peak towards the higher molecular weight end (Figs. 9b and 10a). In the case of the Ecurie sample, however, the organic compounds in the extract appear to be derived from particles of chimney sulfides included in the sample rather than the hydrothermal fluid itself. These observations suggest that the compounds reported by Brault et al. (1988) may also have been derived from chimney particulates entrained into the sampler during fluid collection, which common occurrence for the large syringe samplers used in that study.

\subsection{Abiotic organic compounds}

One of the primary reasons that deep-sea hydrothermal systems have become strong candidates as the site of the origin of life is that conditions within these systems appear to be highly favorable for the abiotic synthesis of organic compounds (e.g., Shock, 1990, 1992; Shock and Schulte, 1998; McCollom and Seewald, 2007). Ultramafic-hosted hydrothermal systems are viewed as particularly favorable environments for abiotic synthesis owing to the strongly reducing conditions and highly elevated concentrations of $\mathrm{H}_{2}$ that develop during serpentinization of ultramafic rocks (Berndt et al., 1996; McCollom and Seewald, 2001, 2013). Indeed, a substantial body of evidence has accumulated that the methane and other light hydrocarbons found in fluids discharging from the ultramafic-hosted deep-sea hydrothermal vents at Lost City and Rainbow have a predominantly abiotic origin (Prokurowski et al., 2008; Charlou et al., 2002, 2010). Formate has also been identified as a product of abiotic carbon reduction at Lost City (Lang et al., 2010).

Whether other, more-complex organic compounds with an abiotic origin can be identified in deep-sea hydrothermal fluids remains an open issue. Holm and Charlou (2001) reported detection of $\mathrm{C}_{16}-\mathrm{C}_{29} n$-alkanes in hydrothermal fluids from the Rainbow system that they attributed to abiotic formation through Fischer-Tropsch-type synthesis in the hydrothermal system. Konn et al. (2009) also speculated that some of the $n$-alkanes and $n$-alkanoic acids they observed in extracts of Lost City and Rainbow hydrothermal fluids might have an abiotic origin.

In the present study, no evidence was found for the presence of abiotic organic compounds in any of the hydrothermal fluid extracts. Although linear alkanes are the primary products of 
Fischer-Tropsch-type abiotic organic synthesis (e.g., McCollom and Seewald, 2007; McCollom et al., 2010), alkanes were observed at only one site during this study (Ecurie at Rainbow) and those compounds were likely derived from particulate matter rather than the fluid. Furthermore, while Fischer-Tropsch products characteristically exhibit a log-linear decrease in abundance with increasing carbon number (e.g., McCollom and Seewald, 2006; McCollom et al. 2010), the alkanes observed at the Ecurie site show a bell-shaped distribution centered at $\sim \mathrm{C}_{28}$ with no compounds below $\mathrm{C}_{23}$ (Figs. 9b and 10a). Thus, the distribution of these hydrocarbons does not resemble the distribution that would be expected for abiotically formed compounds. As suggested by Simoneit et al. (2004), thermal alteration of organic precursors within the hydrothermal system is the most likely source for these compounds. Fischer-Tropsch synthesis also produces alkanoic acids, but the abundance of those compounds would also be expected to follow a regular log-linear decrease with carbon number with no even-odd carbon number preference (e.g., McCollom et al., 1999; McCollom et al. 2010). While alkanoic acids were found in the Lost City fluids and chimney extracts, the strong even-carbon-number preference for these compounds indicates they are biological products.

The absence of extractable abiotic hydrocarbons in the hydrothermal fluids should not necessarily be completely unexpected. Assuming that abiotic hydrocarbons in deep-sea hydrothermal fluids would follow a distribution similar to typical Fischer-Tropsch products, extrapolation from the concentrations of light hydrocarbons observed in the Lost City and Rainbow systems (which are in the nmol kg${ }^{-1}$ range) to predict the expected concentrations of the extractable higher hydrocarbons $\left(\geq \mathrm{C}_{10}\right)$ suggests that these compounds should be present at levels of $1 \mathrm{pmol} \mathrm{kg}^{-1}$ or less (Fig. 12). At such low concentrations, it would be very difficult to detect abiotic hydrocarbons even if they could be concentrated significantly during extraction by SPE or other methods. Other abiotic high-molecular-weight organic compounds (alkanoic acids, etc.) might be expected to have similarly low concentrations, suggesting that it may be extremely challenging to detect the presence of these compounds in deep-sea hydrothermal fluids.

\section{CONCLUDING REMARKS}

The results of this study illustrate many of the difficulties faced in trying to detect solvent extractable hydrocarbons and other higher molecular weight dissolved organic compounds in end-member deep-sea hydrothermal fluids. These compounds, if they are present at all, appear to occur at dissolved concentrations that are at or below the detection limit of commonly used extraction and analysis methods. Additional detection of organic compounds may require use of methods to concentrate the compounds from large amounts of fluid prior to analysis. Furthermore, because of the very low levels at which these compounds occur, it is essential to fully evaluate potential background sources of organic compounds that could easily be mistaken for components dissolved in the fluids, including those from sources such as chimney particulates, biomass, and contaminants introduced during sample handling. The POP gun and SPE sorbent approaches used in this study show some promise for concentrating organic compounds and lowering detection limits, but clearly the level of background compounds from the sorbent phases needs to be significantly reduced to maximize the utility of this method. If this can be accomplished, the ability to concentrate organic compounds from several liters or more of hydrothermal fluids may allow even trace compounds to be detected and characterized, and may ultimately reveal additional details of the sources and fate of organic matter in deep-sea hydrothermal environments. 
954 Acknowledgements - This research was supported by the NSF Ocean Sciences directorate 955 through grants MGG-OCE 0550800 to T.M.M. and MGG-OCE 0549829 to J.S.S. and C.R.G. 956 Additional support for completion of analyses and manuscript preparation was provided by the 957 Alfred P. Sloan Foundation though the Deep Carbon Observatory. The authors thank the captain 958 and crew of the R/V Roger Revelle and the crew of the Jason ROV who made collection of the 959 samples possible. The authors also thank chief scientist Anna-Louise Reysenbach and the other 960 members of the KNOX18RR expedition for their help and cooperation during the cruise. The 961 authors are grateful for helpful comments from three anonymous reviewers and AE Jeff Alt that 962 greatly improved the manuscript.

963 
Barnes I., Rapp J. B., O’Neill J. R., Sheppard R. A., and Gude A. J., III (1972) Metamorphic assemblages and the direction of flow of metamorphic fluids in four instances of serpentinization. Contrib. Mineral. Petrol. 35, 263-276.

Berndt M. E., Allen D. E. and Seyfried W. E., Jr. (1996) Reduction of $\mathrm{CO}_{2}$ during serpentinization of olivine at $300^{\circ} \mathrm{C}$ and 500 bar. Geology 24, 351-354.

Bradley A. S. and Summons R. E. (2009) Multiple origins of methane at the Lost City Hydrothermal Field. Earth Planet. Sci. Lett. 297, 34-41.

Bradley A. S., Hayes J. M., and Summons R. E. (2009) Extraordinary ${ }^{13} \mathrm{C}$ enrichment of diether lipids at the Lost City Hydrothermal Field indicates a carbon-limited ecosystem. Geochim. Cosmochim. Acta 73, 102-118.

Brault M., Simoneit B. R. T., Marty J. C. and Saliot A. (1988) Hydrocarbons in waters and particulate material from hydrothermal environments at the East Pacific Rise, $13^{\circ} \mathrm{N}$. Org. Geochem. 12, 209-219.

Brazelton W. J., Schrenk M. O., Kelley D. S., and Baross J. A. (2006) Methane- and sulfurmetabolizing microbial communities dominate the Lost City hydrothermal field ecosystem. Appl. Environ. Microbiol. 72, 6257-6270.

Charlou J. L., Donval J. P., Jean-Baptiste P., Dapoigny A. and Rona P. A. (1996) Gases and helium isotopes in high temperature solutions sampled before and after ODP Leg 158 drilling at TAG hydrothermal field (26 N MAR). Geophys. Res. Lett. 23, 3491-3494.

Charlou J. L., Donval J. P., Douville E., Jean-Baptiste P., Radford-Knoery J., Fouquet Y., Dapoigny A., and Stievenard M. (2000) Compared geochemical signatures and the

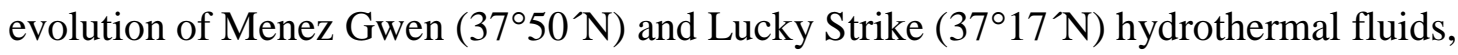
south of the Azores Triple Junction on the Mid-Atlantic Ridge. Chem. Geol. 171, 49-75.

Charlou J. L., Donval J. P., Fouquet Y., Jean-Baptiste P. and Holm N. (2002) Geochemistry of high $\mathrm{H}_{2}$ and $\mathrm{CH}_{4}$ vent fluids issuing from ultramafic rocks at the Rainbow hydrothermal field (36 $14^{\prime}$ N, MAR). Chem. Geol. 191, 345-359.

Charlou J. L., Donval J. P., Konn C., Ondréas H. and Fouquet Y. (2010) High production and fluxes of $\mathrm{H}_{2}$ and $\mathrm{CH}_{4}$ and evidence of abiotic hydrocarbon synthesis by serpentinization in ultramafic-hosted hydrothermal systems on the Mid-Atlantic Ridge. In Diversity of Hydrothermal Systems on Slow-spreading Ocean Ridges (ed. P. Rona, C. Devey, J. Dyment, B. Murton) Geophysical Monograph Series. pp. 265-296.

Delacour A., Früh-Green G. L., Bernasconi S. M., Schaeffer P. and Kelley D. S. (2008) Carbon geochemistry of serpentinites in the Lost City Hydrothermal System $\left(30^{\circ} \mathrm{N}, \mathrm{MAR}\right)$. Geochim. Cosmochim. Acta 72, 3681-3702.

Deming J. W., and Baross J. A. (1993) Deep-sea smokers: Windows to a subsurface biosphere? Geochim. Cosmochim. Acta 57, 3219-3230.

Dittmar T. and Koch B. P. (2006) Thermogenic organic matter dissolved in the abyssal ocean. Mar. Chem. 102, 208-217.

Dittmar T. and Paeng J. (2009) A heat-induced molecular signature in marine dissolved organic matter. Nat. Geosci. 2, 175-179.

Feth J. H., Rogers S. M., and Roberson C. E. (1961) Aqua de Ney, California, a spring of unique chemical character. Geochim. Cosmochim. Acta 22, 75-86.

Foustoukos D. I. and Seyfried W. E., Jr. (2004) Hydrocarbons in hydrothermal vent fluids: The role of chromium-bearing catalysts. Science 304, 1002-1005 
1011

1012

1013

1014

1015

1016

1017

1018

1019

1020

1021

1022

1023

1024

1025

1026

1027

1028

1029

1030

1031

1032

1033

1034

1035

1036

1037

1038

1039

1040

1041

1042

1043

1044

1045

1046

1047

1048

1049

1050

1051

1052

1053

1054

1055

Foustoukos D. I., Savov I. P., and Janecky D. R. (2008) Chemical and isotopic constraints on water/rock interactions at the Lost City hydrothermal field, $30^{\circ} \mathrm{N}$ Mid-Atlantic Ridge. Geochim. Cosmochim. Acta 72, 5457-5474.

Foustoukos D. I., Pester N. J., Ding K., and Seyfried W. E., Jr. (2009) Dissolved carbon species in associated diffuse and focused flow hydrothermal vents at the Main Endeavour Field, Juan de Fuca Ridge: Phase equilibria and kinetic constraints. Geochem. Geophys. Geosyst. 10, Q10003, doi:10.1029/2009GC002472.

Fuchida S., Mizuno Y., Masuda H., Toki T., and Mikita H. (2014) Concentrations and distributions of amino acids in black and white smoker fluids at temperatures over $200{ }^{\circ} \mathrm{C}$. Org. Geochem. 66, 98-106.

German C. R. and Seyfried W. E., Jr. (2014) Hydrothermal Processes. In Treatise on Geochemistry, $2^{\text {nd }}$ Edition (ed. H. D. Holland and K. K.Turekian). Vol. 8, pp. 191-233.

Haberstroh P. R. and Karl D. M. (1989) Dissolved free amino acids in hydrothermal vent habitats of the Guaymas Basin. Geochim. Cosmochim. Acta 53, 2937-2945.

Hertkorn N., Harir M., Koch B. P., Michalke B., and Schmitt-Kopplin P. (2013) High-field NMR spectroscopy and FTICR mass spectrometry: powerful discovery tools for the molecular level characterization of marine dissolved organic matter. Biogosci. 10, 1583-1624.

Holm N. G., and Charlou J. L. (2001) Initial indications of abiotic formation of hydrocarbons in the Rainbow ultramafic hydrothermal system, Mid-Atlantic Ridge. Earth Planet. Sci. Lett. 191, 1-8.

Horiuchi T., Takano Y., Ishibashi J., Marumo K., Urabe T., and Kobayashi K. (2004) Amino acids in water samples from deep sea hydrothermal vents at Suiyo Seamount, Izu-Bonin Arc, Pacific Ocean. Org. Geochem. 35, 1121-1128.

Huang Y., Wang Y., Alexandre M. R., Lee T., Rose-Petruck C., Fuller M., and Pizzarello S. (2005) Molecular and compound-specific isotopic characterization of monocarboxylic acids in carbonaceous chondrites. Geochim. Cosmochim. Acta 69, 1073-1084.

Kawka O. E., and Simoneit B. R. T. (1994) Hydrothermal pyrolysis of organic matter in Guaymas Basin: 1. Comparison of hydrocarbon distributions in subsurface sediments and seabed petroleums. Org. Geochem. 22, 947-978.

Kelley D. S., Karston J. A., Früh-Green G. L., Yoerger D. R., Shank T. M., Butterfield D. A., Hayes J. M., Schrenk M. O., Olson E. J., Proskurowski G, Jakuba M., Bradley A., Larson B., Ludwig K., Glickson D., Buckman K., Bradley A. S., Brazelton W. J., Roe K., Elend M. J., Delacour A., Bernasconi S. M., Lilley M. D., Baross J. A., Summons R. E., Sylva S. P. (2005) A serpentinite-hosted ecosystem: The Lost City hydrothermal field. Science 307, 1428-1434.

Klevenz V., Sumoodur A., Ostertag-Henning C., and Koschinsky A. (2010) Concentrations and distributions of dissolved amino acids in fluids from Mid-Atlantic Ridge hydrothermal vents. Geochem. J. 44, 387-397.

Konn C., Charlou J. L., Donval J. P., Holm N. G., Dehairs F. and Bouillon S. (2009) Hydrocarbons and oxidized organic compounds in hydrothermal fluids from Rainbow and Lost City ultramafic-hosted vents. Chem. Geol. 258, 299-314.

Konn C., Charlou J. L., Donval J. P., and Holm N. G. (2012) Characterisation of dissolved organic compounds in hydrothermal fluids by stir bar sorptive extraction-gas chromatography-mass spectrometry. Case study: the Rainbow field ( $36^{\circ} \mathrm{N}$, Mid-Atlantic Ridge). Geochem. Transact. 13:8. 
1056

1057

1058

1059

1060

1061

1062

1063

1064

1065

1066

1067

1068

1069

1070

1071

1072

1073

1074

1075

1076

1077

1078

1079

1080

1081

1082

1083

1084

1085

1086

1087

1088

1089

1090

1091

1092

1093

1094

1095

1096

1097

1098

1099

1100

Lang S. Q., Butterfield D. A., Schulte M., Kelley D. S., and Lilley M. D. (2010) Elevated concentrations of formate, acetate and dissolved organic carbon found at the Lost City hydrothermal field. Geochim. Cosmochim. Acta 74, 941-952.

Lang, S. Q., Früh-Green G. L., Bernasconi S. M., Lilley M. D., Proskurowski G., Méhay S., and Butterfield D. A. (2012) Microbial utilization of abiogenic carbon and hydrogen in a serpentinite-hosted system. Geochim. Cosmochim. Acta 92, 82-99.

Lang S. Q., Früh-Green G. L., Bernasconi S. M., and Butterfield D. A. (2013) Sources of organic nitrogen at the serpentinite-hosted Lost City hydrothermal field. Geobiology 11, 154-169.

Legras J. L., Erny C., Le Jeune C., Lollier M., Adolphe Y., Demuyter C., Delobel P., Blondin B., and Karst F. (2010) Activation of two different resistance mechanisms in Saccharomyces cerevisiae upon exposure to octanoic and decanoic acids. Appl. Environ. Microbiol. 76, 7526-7535.

Lilley, M. D., J. A. Baross, and L. I. Gordon (1983) Reduced gases and bacteria in hydrothermal fluids: The Galapagos Spreading Center and $21^{\circ} \mathrm{N}$ East Pacific Rise. In Hydrothermal Processes at Seafloor Spreading Centers (ed. P. A. Rona et al.) Plenum Press. pp. 411-447.

Lilley M. D., Butterfield D. A., Olson E. J., Lupton J. E., Macko S. A., and McDuff R. E. (1993) Anomalous $\mathrm{CH}_{4}$ and $\mathrm{NH}_{4}{ }^{+}$concentrations at an unsedimented mid-ocean-ridge hydrothermal system. Nature J2-364, 45-47.

Martens C. S. (1990) Generation of short chain organic acid anions in hydrothermally altered sediments of the Guaymas Basin, Gulf of California. Appl. Geochem. 5, 71-76.

McCollom T. M. and Seewald J. S. (2001) A reassessment of the potential for reduction of dissolved $\mathrm{CO}_{2}$ to hydrocarbons during serpentinization of olivine. Geochim. Cosmochim. Acta 65, 3769-3778.

McCollom T. M. and Seewald J. S. (2006) Carbon isotope composition of organic compounds produced by abiotic synthesis under hydrothermal conditions. Earth Planet. Sci. Lett. 243, 74-84.

McCollom, T.M., and Seewald J. S. (2007) Abiotic synthesis of organic compounds in deep-sea hydrothermal environments. Chem. Rev., 107, 382-401.

McCollom T. M. and Seewald J. S. (2013) Serpentinites, hydrogen, and life. Elements, 9, 129134.

McCollom T. M., Ritter G., and Simoneit B. R. T. (1999) Lipid synthesis under hydrothermal conditions by Fischer-Tropsch-type reactions. Origins Life Evol. Biosphere 29, 153-166.

McCollom T. M., Sherwood Lollar B, Lacrampe-Couloume G. and Seewald, J. S. (2010) The influence of carbon source on abiotic organic synthesis and carbon isotope fractionation under hydrothermal conditions. Geochim. Cosmochim. Acta 74, 2717-2740.

Méhay S., Früh-Green G. L., Lang S. Q., Bernasconi S. M., Brazelton W. J., Schrenck M. O., Schaeffer P. and Adam P. (2013) Record of archaeal activity at the serpentinite-hosted Lost City Hydrothermal Field. Geobiology 11, 570-592.

Pasini V., Brunelli D., Dumas P., Sandt C., Frederick J., Benzerara, Bernard S., and Ménez B. (2013) Low temperature hydrothermal oil and associated biological precursors in serpentinites from Mid-Ocean Ridge. Lithos 178, 84-95.

Proskurowksi G., Lilley M. D., Kelley D. S., and Olson E. J. (2006) Low temperature volatile production at the Lost City Hydrothermal Field, evidence from a hydrogen stable isotope geothermometer. Chem. Geol. 229, 331-343. 
Proskurowksi G., Lilley M. D., Seewald J. S., Früh-Green G. L., Olson E. J., Lupton J. E.,

Hydrothermal Field. Science 319, 604-607

Reeves E. P., McDermott J. M., and Seewald J. S. (2014) The origin of methanethiol in midocean ridge hydrothermal fluids. Proc. Nat. Acad, Sci. USA 111, 5474-5479.

Schrenk M. O., Kelley D. S., Bolton S. A., and Baross J. A. (2006) Low archaeal diversity linked to subseafloor geochemical processes at the Lost City hydrothermal filed, MidAtlantic Ridge. Environ. Microbiol. 6, 1086-1095.

Seewald J. S., Doherty K. W., Hammar T. R., and Liberatore S. P. (2002) A new gas-tight isobaric sampler for hydrothermal fluids. Deep-Sea Res. I 49, 189-196.

Seyfried W. E., Jr., Foustoukos D. I. and Fu. Q. (2007) Redox evolution and mass transfer during serpentinization: An experimental and theoretical study at $200^{\circ} \mathrm{C}, 500$ bar with implications for ultramafic-hosted hydrothermal systems at Mid-Ocean Ridges. Geochim. Cosmochim. Acta 71, 3872-3886.

Seyfried W. E., Jr., Foustoukos D. I. and Fu. Q. (2011) Vent fluid chemistry of the Rainbow hydrothermal system $\left(36^{\circ} \mathrm{N}, \mathrm{MAR}\right)$ : Phase equilibria and in situ $\mathrm{pH}$ controls on subseafloor alteration processes. Geochim. Cosmochim. Acta 75, 1574-1593.

Shock E. L. (1990) Geochemical constraints on the origin of organic compounds in hydrothermal systems. Origins Life Evol. Biospheres 20, 331-367.

Shock E. L. (1992) Chemical environments of submarine hydrothermal systems, Origins Life Evol. Biospheres 22, 67-107.

Shock E. L., and Schulte M. D. (1998) Organic synthesis during fluid mixing in hydrothermal systems. J. Geophys. Res. 103, 28,513-28,517.

Simoneit B. R. T. (1988) Petroleum generation in submarine hydrothermal systems: An update. Can. Mineral. 26, 827-840.

Simoneit B. R. T., Lein A.Yu., Peresypkin V. I., and Osipov G. A. (2004) Composition and origin of hydrothermal petroleum and associated lipids in the sulfide deposits of the Rainbow Field (Mid-Atlantic Ridge at $36^{\circ} \mathrm{N}$ ). Geochim. Cosmochim. Acta 68, 2275-2294.

Ventura G. T., Simoneit B. R. T., Nelson R. K., and Reddy C. M. (2012) The composition, origin and fate of complex mixtures in the maltene fractions of hydrothermal petroleum assessed by comprehensive two-dimensional gas chromatography. Org. Geochem. 45, 48-65.

Von Damm K. L., Bray A. M., Buttermore L. G., and Oosting S. E. (1998) The geochemical controls on vent fluids from the Lucky Strike vent field, Mid-Atlantic Ridge. Earth Planet. Sci. Lett. 160, 521-536.

Welhan J. A. and Craig H. (1983) Methane, hydrogen, and helium in hydrothermal fluids at $21^{\circ} \mathrm{N}$ on the East Pacific Rise. In Hydrothermal Processes at Seafloor Spreading Centers ( ed. P. A. Rona, et al.), pp. 391-409. Plenum Press.

Welhan J. A. and Lupton J. E. (1987) Light-hydrocarbon gases in Guaymas Basin hydrothermal fluids-Thermogenic versus abiogenic origin. Bull. Am. Assoc. Petrol. Geol., 71, 215-223.

Yuen G. U. and Kvenvolden K. A. (1973) Monocarboxylic acids in Murray and Murchison carbonaceous meteorites. Science 246, 301-303. 
Table 1. Summary of samples collected for analysis of organic compounds, with selected chemical information.

\begin{tabular}{|c|c|c|c|c|c|c|c|c|c|c|c|}
\hline \multirow[t]{2}{*}{ Site/Vent } & \multirow{2}{*}{$\begin{array}{l}\text { Temp. } \\
\left({ }^{\circ} \mathrm{C}\right)\end{array}$} & \multirow{2}{*}{$\begin{array}{l}\text { Lat. } \\
(\mathrm{N})\end{array}$} & \multirow{2}{*}{$\begin{array}{l}\text { Long. } \\
\text { (W) }\end{array}$} & \multicolumn{3}{|c|}{ - Sample ID } & \multirow{2}{*}{$\begin{array}{c}\mathrm{pH} \\
\left(25^{\circ} \mathrm{C}\right)\end{array}$} & \multirow[t]{2}{*}{$\mathrm{Mg}$} & \multirow{2}{*}{$\mathrm{H}_{2}$} & \multirow[t]{2}{*}{$\Sigma \mathrm{CO}_{2}$} & \multirow[t]{2}{*}{$\mathrm{CH}_{4}$} \\
\hline & & & & IGT & POP gun & SPE(phase) & & & & & \\
\hline \multicolumn{12}{|c|}{ Rainbow (ultramafic-hosted) } \\
\hline Guillaume & 359 & $36^{\circ} 13.752^{\prime}$ & $33^{\circ} 54.222^{\prime}$ & J2-352-IGT7 & $\begin{array}{l}\text { J2-352-POP1, } \\
\text { J2-352-POP2 }\end{array}$ & & 3.24 & 1.9 & 15.8 & 21.2 & 2.03 \\
\hline Regner & 326 & $36^{\circ} 13.751^{\prime}$ & $33^{\circ} 54.232^{\prime}$ & J2-353-IGT7 & & & 3.45 & 8.0 & 13.5 & 21.1 & 1.86 \\
\hline Padraig & 350 & $36^{\circ} 13.766^{\prime}$ & $33^{\circ} 54.173^{\prime}$ & J2-353-IGT6 & $\begin{array}{l}\text { J2-353-POP1, } \\
\text { J2-353-POP2 }\end{array}$ & & 3.43 & 5.4 & 16.1 & 19.6 & 1.79 \\
\hline Stylo 1 & 366 & $36^{\circ} 13.781^{\prime}$ & $33^{\circ} 54.070^{\prime}$ & J2-354-IGT7 & & & 3.28 & 2.1 & 15.0 & 24.6 & 2.10 \\
\hline Ecurie & 191 & $36^{\circ} 13.748^{\prime}$ & $33^{\circ} 54.188^{\prime}$ & J2-354-IGT6 & $\begin{array}{l}\mathrm{J} 2-354-\mathrm{POP} 1 \\
\mathrm{~J} 2-354-\mathrm{POP} 2\end{array}$ & J2-354-SPE(HLB) & 2.99 & 7.4 & 9.4 & 18.7 & 1.79 \\
\hline Stylo 2 & 359 & $36^{\circ} 13.777^{\prime}$ & $33^{\circ} 54.072^{\prime}$ & J2-355-IGT7 & $\begin{array}{l}\text { J2-355-POP1, } \\
\text { J2-355-POP2 }\end{array}$ & $\begin{array}{l}\text { J2-355-SPE(C8), } \\
\text { J2-355-SPE(HLB) }\end{array}$ & 3.27 & 2.6 & 16.5 & 20.9 & 2.10 \\
\hline \multicolumn{12}{|c|}{ Lucky Strike (basalt-hosted) } \\
\hline 2608(top) & 323 & $37^{\circ} 17.508^{\prime}$ & $32^{\circ} 16.675^{\prime}$ & J2-356-IGT6 & & & 3.86 & 0.9 & 0.048 & 34.7 & 1.01 \\
\hline US4 & 299 & $37^{\circ} 17.276^{\prime}$ & $32^{\circ} 16.529^{\prime}$ & J2-357-IGT6 & & & 4.75 & 29.5 & 0.053 & 133 & 0.74 \\
\hline Crystal & 306 & $37^{\circ} 17.450^{\prime}$ & $32^{\circ} 16.920^{\prime}$ & J2-358-IGT6 & & & 3.64 & 1.5 & 0.041 & 117 & 0.82 \\
\hline Medea & 251 & $37^{\circ} 17.659^{\prime}$ & $32^{\circ} 16.900^{\prime}$ & J2-359-IGT6 & $\mathrm{J} 2-359-\mathrm{POP} 1^{\#}$ & J2-359-SPE(HLB) ${ }^{\#}$ & 3.68 & 2.2 & 0.063 & 98 & 0.89 \\
\hline \multicolumn{12}{|c|}{ Lost City (ultramafic-hosted) } \\
\hline Beehive & 91 & $30^{\circ} 7.431^{\prime}$ & $42^{\circ} 7.203^{\prime}$ & J2-360-IGT6 & & & 10.6 & 0.1 & 10.4 & 0.18 & 1.9 \\
\hline Beehive & 90 & $30^{\circ} 7.434^{\prime}$ & $42^{\circ} 7.202^{\prime}$ & J2-361-IGT6 & $\begin{array}{l}\text { J2-361-POP1, } \\
\text { J2-361-POP2 }\end{array}$ & $\begin{array}{l}\text { J2-361-SPE(HLB), } \\
\text { J2-361-SPE(C8) }\end{array}$ & 10.6 & 1.0 & 10.4 & 0.18 & 1.9 \\
\hline Top of Poseidon & 51 & $30^{\circ} 7.430^{\prime}$ & $42^{\circ} 7.203^{\prime}$ & J2-362-IGT6 & & & 10.8 & 2.6 & - & - & - \\
\hline \multicolumn{4}{|c|}{ Seawater (Lucky Strike) } & & J2-356-POP1 & J2-356-SPE(HLB) & - & - & - & - & - \\
\hline \multicolumn{4}{|c|}{ Seawater (Lost City) } & & J2-362-POP1 & J2-362-SPE(C8) & - & - & - & - & - \\
\hline \multicolumn{4}{|c|}{ Seawater (TAG) } & J2-364-IGT6 & & & - & - & - & - & - \\
\hline
\end{tabular}

Dissolved concentrations of $\mathrm{Mg}, \mathrm{H}_{2}, \Sigma \mathrm{CO}_{2}$, and $\mathrm{CH}_{4}$ in mmol kg${ }^{-1} . \Sigma \mathrm{CO}_{2}$ is total dissolved $\mathrm{CO}_{2}\left(=\mathrm{CO}_{2(a q)}+\mathrm{HCO}_{3}{ }^{-}+\mathrm{CO}_{3}{ }^{2-}\right)$. “-“ $=$ not measured. ${ }^{\#}$ For this sample, fluid was processed through the POP gun for only 20 minutes; for all other POP gun samples, fluids were pumped for about 60 minutes. 
Table 2. Summary of organic compounds detected in hydrothermal vent fluid samples.

\begin{tabular}{|c|c|c|c|c|c|c|c|c|c|c|c|c|}
\hline \multirow[b]{2}{*}{$\begin{array}{c}\text { Site: } \\
\text { Sample types: }\end{array}$} & \multicolumn{6}{|c|}{ Rainbow } & \multicolumn{4}{|c|}{ Lucky Strike } & \multicolumn{2}{|c|}{ Lost City } \\
\hline & $\begin{array}{c}\text { Guillaume } \\
\text { (I,P,S) }\end{array}$ & $\begin{array}{l}\text { Regner } \\
\text { (I) }\end{array}$ & $\begin{array}{c}\text { Padraig } \\
(\mathrm{I}, \mathrm{P}, \mathrm{S})\end{array}$ & $\begin{array}{l}\text { Stylo1 } \\
\text { (I) }\end{array}$ & $\begin{array}{l}\text { Ecurie } \\
(\mathrm{I}, \mathrm{P}, \mathrm{S})\end{array}$ & $\begin{array}{l}\text { Stylo2 } \\
(\mathrm{I}, \mathrm{P}, \mathrm{S})\end{array}$ & $\begin{array}{c}2608 \\
(\mathrm{I})\end{array}$ & $\begin{array}{l}\text { US4 } \\
\text { (I) }\end{array}$ & $\begin{array}{l}\text { Crystal } \\
\text { (I) }\end{array}$ & $\begin{array}{l}\text { Medea } \\
(I, P, S)\end{array}$ & $\begin{array}{c}\text { Beehive } \\
(\mathrm{I}, \mathrm{P}, \mathrm{S})\end{array}$ & $\begin{array}{c}\text { Poseidon } \\
\text { (I) }\end{array}$ \\
\hline \multicolumn{13}{|l|}{ Carboxylic acids } \\
\hline Octanoic acid & - & - & - & - & - & - & - & - & - & - & $\mathrm{P}$ & $\mathrm{P}$ \\
\hline Decaonoic acid & - & - & - & - & - & - & - & - & - & - & $\mathrm{P}$ & $\mathrm{P}$ \\
\hline Dodecanoic acid & - & - & - & - & - & - & - & - & - & - & $\mathrm{P}$ & $\mathrm{P}$ \\
\hline Tetradecanoic acid & - & - & - & - & - & - & - & - & - & B & - & - \\
\hline Hexadecanoic acid & - & - & - & - & - & - & - & - & - & B & - & - \\
\hline Hexadecenoic acid & - & - & - & - & - & - & - & - & - & B & - & - \\
\hline Octadecanoic acid & - & - & - & - & - & - & - & - & - & B & - & - \\
\hline Octadecenoic acid & $\mathrm{P}$ & - & - & - & - & - & - & - & - & B & - & - \\
\hline Doeicosanoic acid & - & - & - & - & - & - & - & - & - & B & - & - \\
\hline \multicolumn{13}{|l|}{ Aliphatic hydrocarbons } \\
\hline n-Alkanes [range] & - & - & - & - & $\begin{array}{c}\mathrm{CS} \\
{\left[\mathrm{C}_{23}-\mathrm{C}_{33}\right]}\end{array}$ & - & - & - & - & - & - & - \\
\hline Squalene & - & - & - & - & - & - & - & - & - & - & - & $\mathrm{P}$ \\
\hline \multicolumn{13}{|l|}{ Aromatic hydrocarbons } \\
\hline Phenanthrene & $\mathrm{P}$ & - & $\mathrm{P}$ & - & $\mathrm{P}$ & $\mathrm{P}$ & - & - & - & - & - & - \\
\hline Methylphenanthrenes & $\mathrm{P}$ & - & $\mathrm{P}$ & - & $\mathrm{P}$ & $\mathrm{P}$ & - & - & - & - & - & - \\
\hline $\mathrm{C}_{2}$-phenanthrenes & - & - & $\mathrm{P}$ & - & $\mathrm{P}$ & $\mathrm{P}$ & - & - & - & - & - & - \\
\hline Methylfluorene & $\mathrm{P}$ & - & - & - & - & - & - & - & - & - & - & - \\
\hline Dibenzothiophene & - & - & - & - & - & - & - & - & $\mathrm{P}$ & $\mathrm{P}$ & - & - \\
\hline \multicolumn{13}{|l|}{ Other compounds } \\
\hline Cholesterol & - & - & - & - & - & - & - & - & - & - & - & $\mathrm{P}$ \\
\hline Hexadecanol & $\mathrm{P}$ & - & - & - & - & - & - & - & - & - & - & $\mathrm{P}$ \\
\hline Trithiolane & - & - & - & - & - & - & - & - & - & - & - & $\mathrm{P}$ \\
\hline UCM & - & - & - & - & $\mathrm{CS}$ & $\mathrm{P}$ & - & - & - & - & - & - \\
\hline
\end{tabular}

Compounds that were positively identified and inferred to be indigenous to the fluid are designated with a "P", while compounds that were detected but interpreted to come from other sources are identified designated with a "CS" (chimney sulfide source) or "B" (biomass source). "_“= compound not detected in fluid sample. Sample types: I = IGT sampler, P = POP gun, $\mathrm{S}=$ SPE cartridge. 
Table 3. Abundances ${ }^{\dagger}$ and isotopic composition of $n$-alkanoic acids in Lost City hydrothermal fluids samples.

\begin{tabular}{|c|c|c|c|}
\hline Sample & $\begin{array}{c}\text { J2-360- } \\
\text { IGT6 }\end{array}$ & $\begin{array}{l}\text { J2-361- } \\
\text { IGT6 }\end{array}$ & $\begin{array}{c}\text { J2-361- } \\
\text { SPE(HLB) }\end{array}$ \\
\hline & & $\mu \mathrm{mol}$ acid/ & 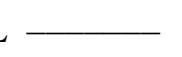 \\
\hline $\mathrm{C}_{8}$ & 0.72 & 0.53 & 0.77 \\
\hline $\mathrm{C}_{10}$ & 0.47 & 0.37 & 0.54 \\
\hline $\mathrm{C}_{12}$ & 0.28 & 0.23 & 0.31 \\
\hline $\mathrm{C}_{8}$ & 5.7 & 4.2 & 6.2 \\
\hline $\mathrm{C}_{10}$ & 4.7 & 3.7 & 5.4 \\
\hline $\mathrm{C}_{12}$ & 3.4 & 2.7 & 3.7 \\
\hline Total & 13.8 & 10.6 & 15.3 \\
\hline $\mathrm{C}_{8}$ & -5.9 & -7.6 & \\
\hline $\mathrm{C}_{10}$ & -4.3 & -2.8 & \\
\hline $\mathrm{C}_{12}$ & -4.8 & -3.6 & \\
\hline
\end{tabular}

${ }^{\dagger}$ Concentrations are given for the acids and for the equivalent number of carbon atoms. 

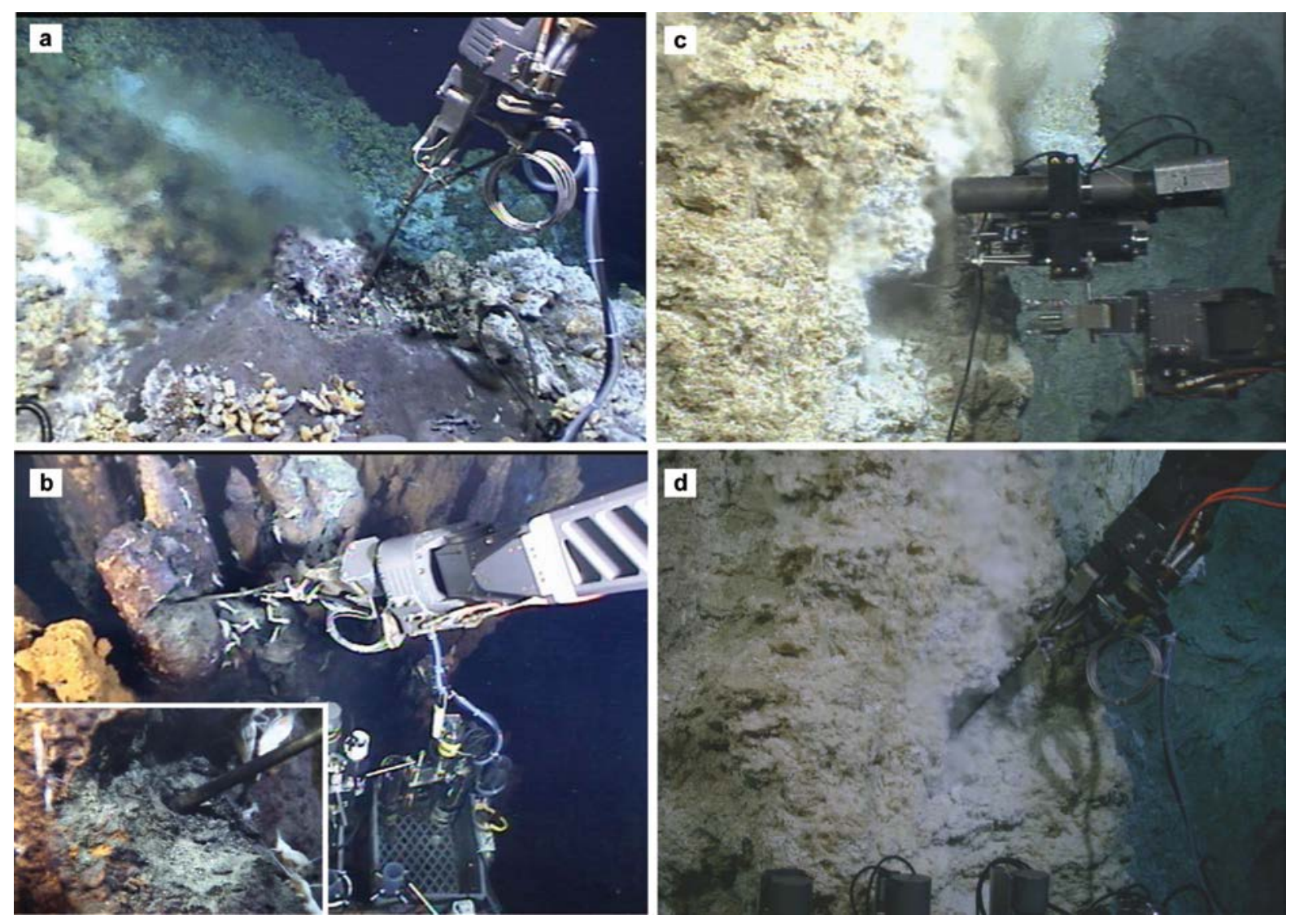

Figure 1. Representative images of fluid sampling at the deep-sea hydrothermal vent sites. (a) Sampling with the POP gun at the Medea site at Lucky Strike. (b) Sampling with the POP gun at the Ecurie site at Rainbow. The fluid at this site was obtained from a cavity excavated into the side of an older, less active chimney structure. Inset shows closeup of tip of POP gun in side of chimney. (c, d) Fluid sampling at the Beehive site at Lost City with the isobaric gas-tight sampler (c) and POP gun (d). 


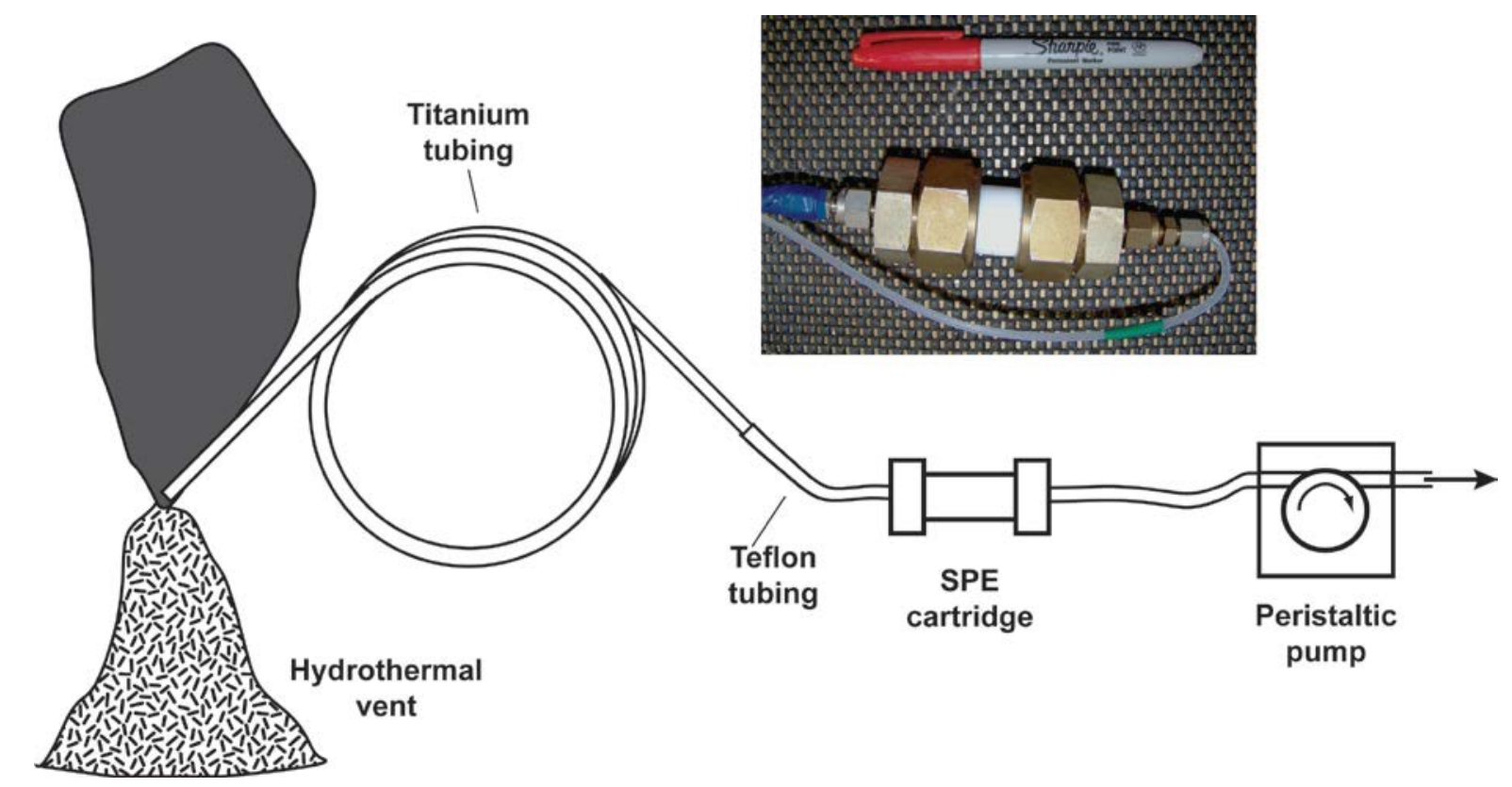

Figure 2. Schematic drawing of the peristaltic organic pump fluid sampling device (POP gun). The sampler consists of a coil of titanium tubing ( $4 \mathrm{~m}$ long) connected through Teflon tubing to a cartridge filled with solid phase extraction (SPE) microbeads, which is then connected to a peristaltic pump with silicon tubing. During operation, the tip of the sampler is placed in a hydrothermal vent, and the peristaltic pump draws fluid through the tubing and SPE cartridge. The inset shows an image of the SPE cartridge, consisting of SPE microbeads within a $2.54 \mathrm{~cm}$ diameter Teflon tube capped with porous Teflon frits and sealed at the ends with stainless steel Swagelock fittings. 


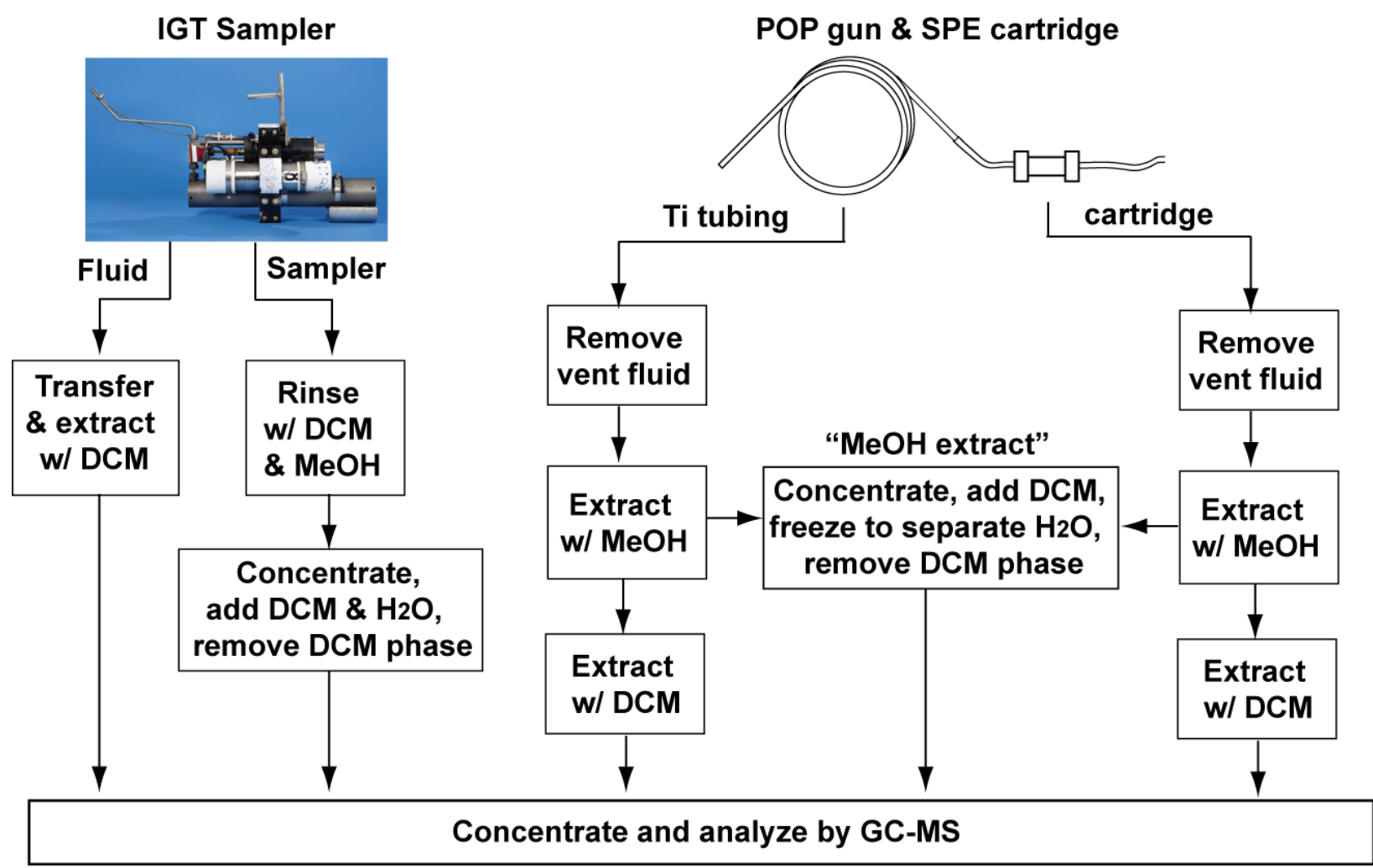

Figure 3. Flow diagram summarizing methods used to extract and prepare organic compounds for analysis from the different sampling methods used in this study. 

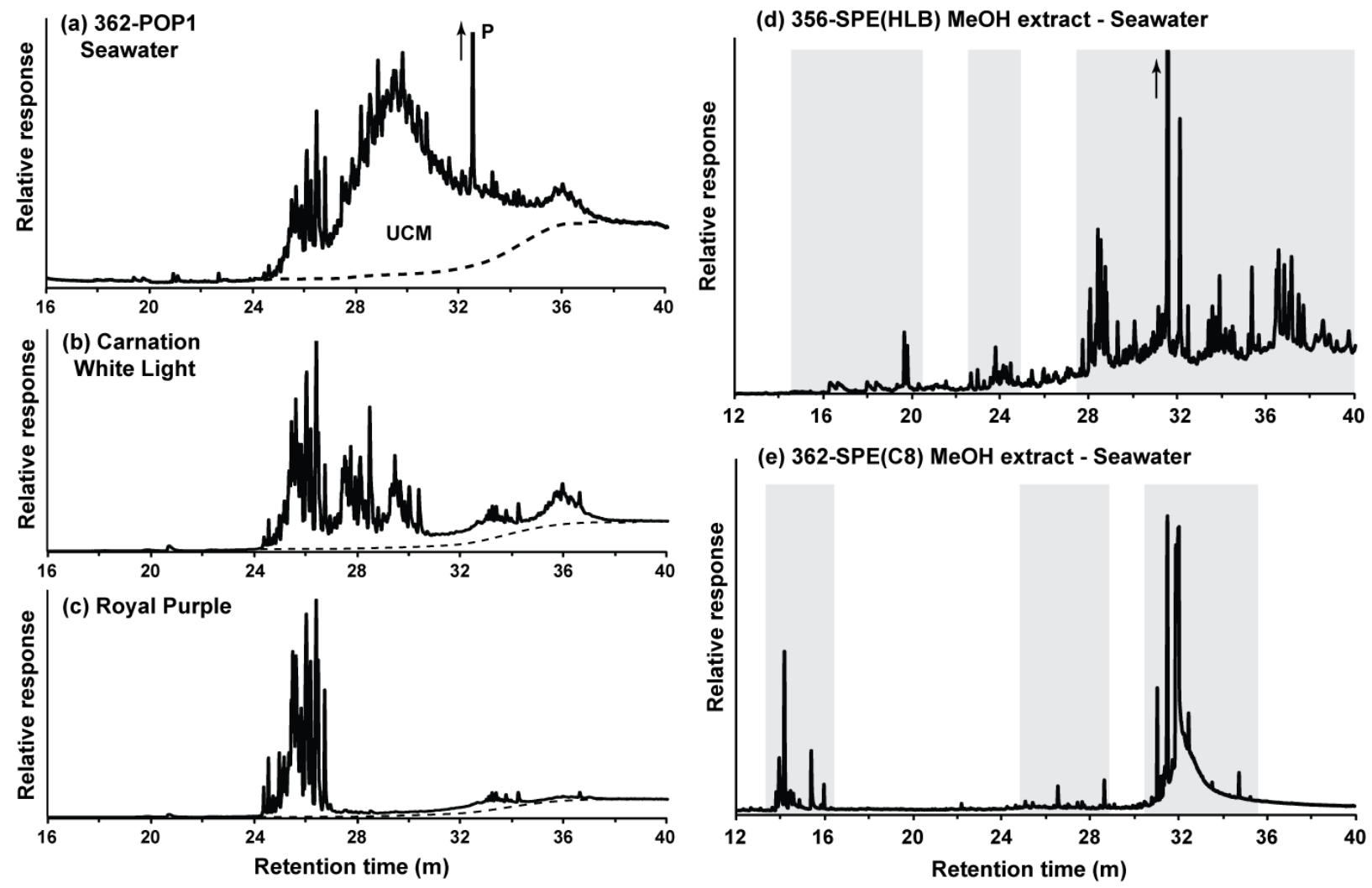

Figure 4. Total ion chromatograms from GC-MS analysis of potential sources of background organic compounds that could have contaminated the hydrothermal fluid samples. (a) $\mathrm{MeOH}$ extract of seawater sample from the vicinity of the TAG hydrothermal field obtained with the POP gun, showing a large unresolved complex mixture of compounds (UCM). Results for a seawater sample taken in the vicinity of the Lucky Strike hydrothermal vent field (J2-356-POP1) were very similar. (b,c) Oils used for lubrication and pressure compensation on ROV Jason. These oils also display a UCM, but they are distinctly different from the seawater UCM. (d,e) $\mathrm{MeOH}$ extracts of deep seawater samples processed using the HLB and C8 SPE cartridges. Gray areas highlight prominent peaks for organic compounds that were present in all samples processed through these cartridges, including Fisher $\mathrm{H}_{2} \mathrm{O}$ blanks, and are inferred to be contaminants extracted from the SPE microbeads. In all but a few cases, the mass fragmentation patterns for these compounds did not match any compounds in the reference database and could not be identified. 

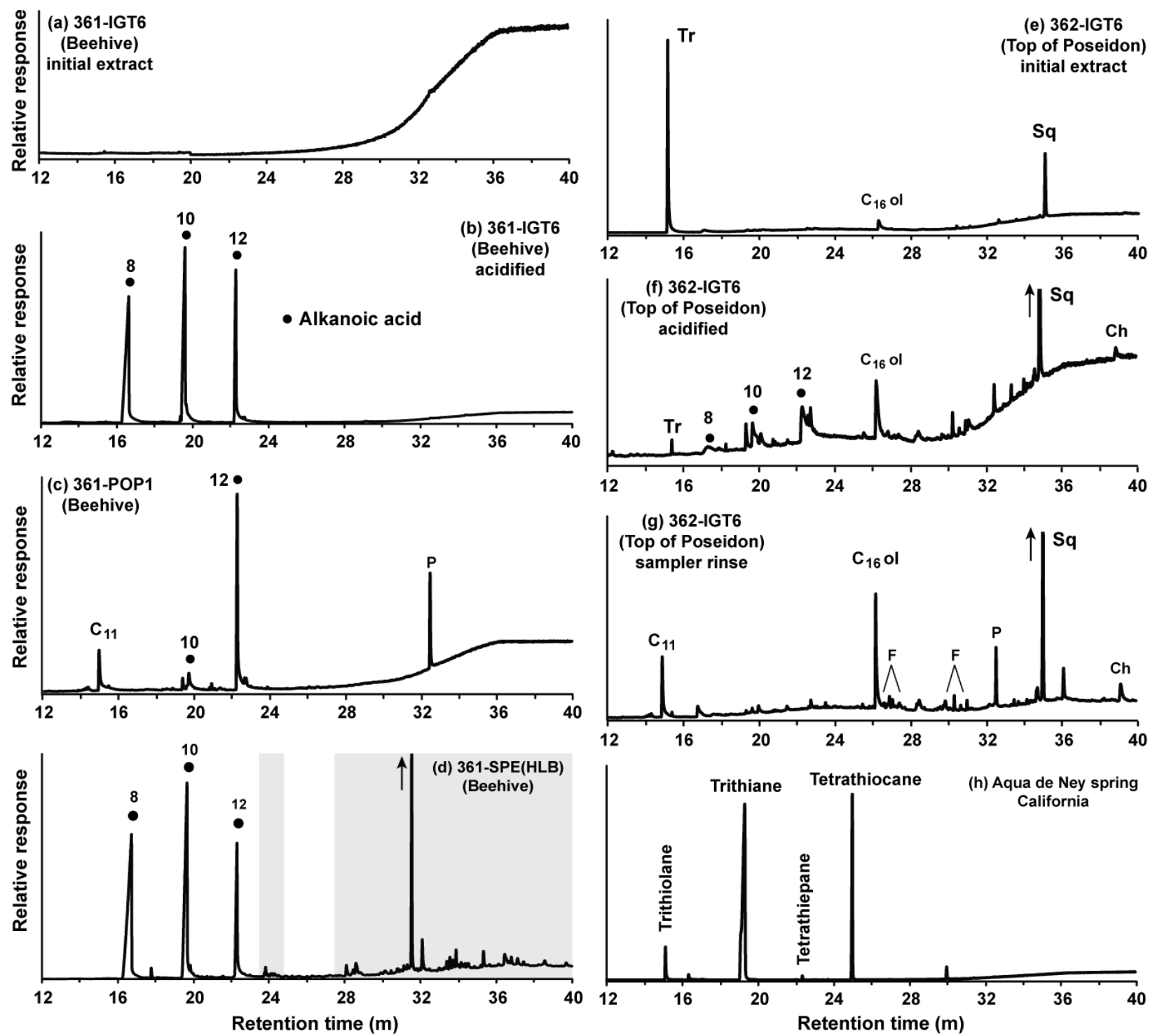

Figure 5. Total ion chromatograms for samples from the Lost City hydrothermal system. (a,e) Initial DCM extracts for samples from Beehive and Top of Poseidon (ToP). The Beehive sample shows no peaks above the instrumental baseline, while the ToP sample has peaks for trithiolane (Tr), hexadecanol $\left(\mathrm{C}_{16} \mathrm{Ol}\right)$, and squalene $(\mathrm{Sq})$. (b,f) Second DCM extracts from Beehive and ToP samples following acidification of the fluid. The Beehive sample exhibits prominent peaks for octanoic, decanoic and dodecanoic acids $\left(\mathrm{C}_{8}, \mathrm{C}_{10}\right.$ and $\mathrm{C}_{12}$, respectively), which are also evident in smaller amounts in the ToP sample along with cholesterol (Ch). Sample J2-360-IGT6 produced results identical to the Beehive sample shown. (c) Analysis of POP gun MeOH extract from Beehive site. The other POP gun sample for this site produced the same result. (d) Sample from Beehive vent processed with the HLB SPE cartridge and extracted with MeOH. Peaks outlined by gray areas are inferred to be contaminants because they were present in all $\mathrm{MeOH}$ extracts of the HLB cartridges (see Fig. 4d and Supplemental Fig. S1). (g) Solvent rinse of IGT sampler from ToP site. (h) Analysis of DCM extract of highly alkaline fluid $(\mathrm{pH}=11.8)$ discharged from Aqua de Ney spring, northern California. The spring fluid includes trithiolane and several other cyclic C-S compounds (trithiane, tetrathiepane, tetrathiocane). The sample was 
collected in a glass bottle and extracted using the same procedures as the IGT samples. Undecane $\left(\mathrm{C}_{11}\right)$ comes from the methanol solvent, compounds labeled " $F$ " are from the Fluorolube lubricant, and "P” is dioctyl phthalate. Unlabeled peaks could not be identified. 

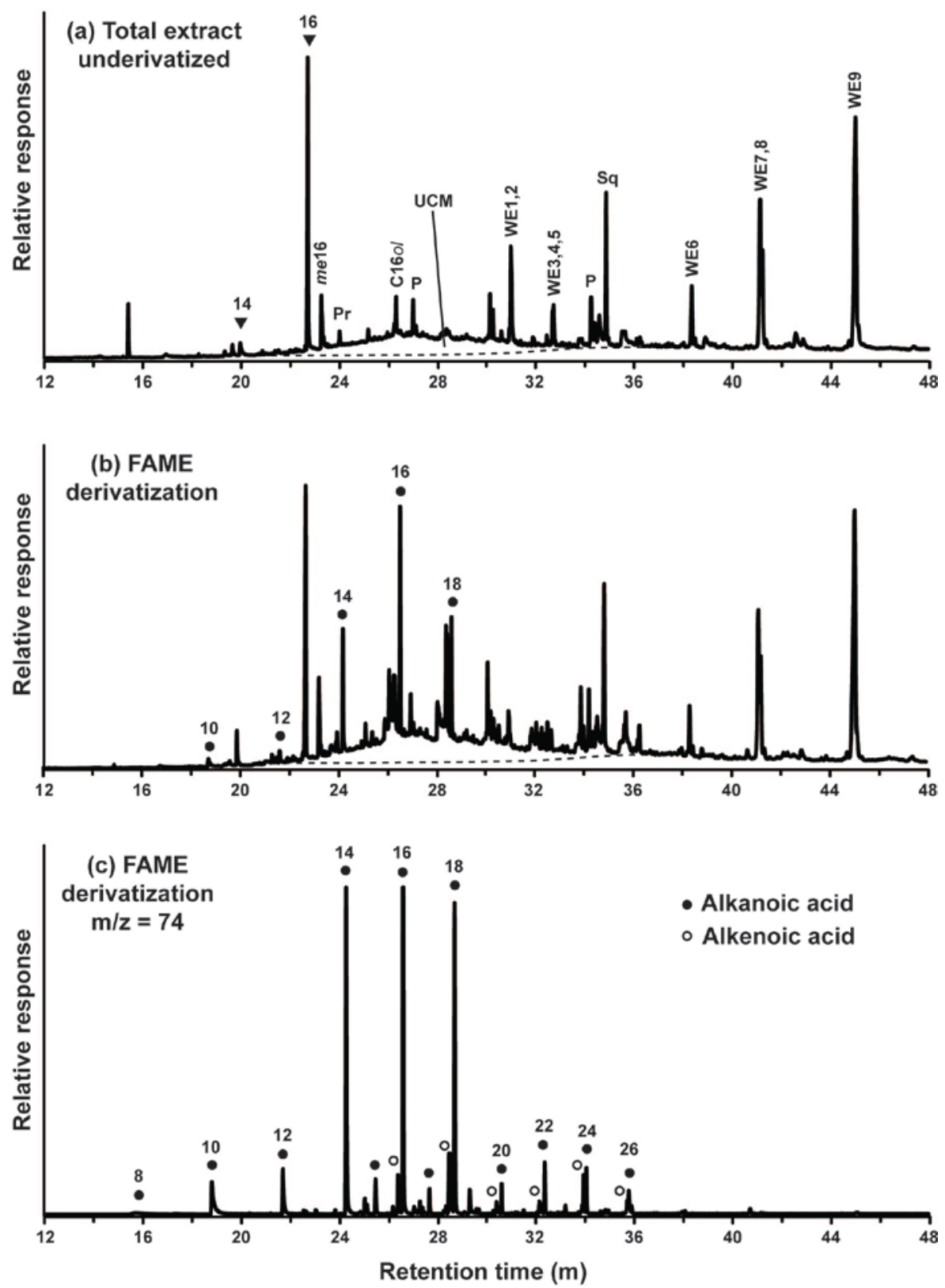

Figure 6. Chromatograms of Lost City chimney extracts. (a) Total ion chromatogram for analysis of total extract. Dashed line shows instrument baseline, and area marked UCM is an unresolved complex mixture of compounds. Peaks labeled "WE" are wax esters, and other labeled peaks are $n$-alkanes (inverted triangles, labeled with carbon number), methylhexadecane (me16), pristane (Pr), hexadecaol (C16ol), and squalene (Sq). (b) Total ion chromatogram of total extract derivatized to convert fatty acids to methyl esters (FAME). Only peaks for methyl esters of the $\mathrm{C}_{10}-\mathrm{C}_{18}$ alkanoic acids are labeled; other peaks correspond to those labeled in (a) and (c). (c) Mass fragmentogram for mass-to-charge ratio (m/z) of 74, characteristic for FAME. 
Labels identify methyl esters of $n$-alkanoic and $n$-alkenoic acids, with number of carbon atoms specified for even-numbered compounds. Alkenoic acids elute just before alkanoic acid of same carbon number (positions of double bonds were not determined). "P" = phthalates. Other abbreviations: WE1,2 $=\mathrm{C}_{10}-\mathrm{C}_{12}$ ester $+\mathrm{C}_{8}-\mathrm{C}_{14}$ ester; WE3,4,5 $=\mathrm{C}_{12}$ - $\mathrm{C}_{12}$ ester $+\mathrm{C}_{10}-\mathrm{C}_{14}$ ester + $\mathrm{C}_{8}-\mathrm{C}_{14}$ ester; WE6 $=\mathrm{C}_{14}-\mathrm{C}_{16}$ ester; WE7,8 $=\mathrm{C}_{14}-\mathrm{C}_{18}$ ester $+\mathrm{C}_{16}-\mathrm{C}_{16}$ ester; WE9 $=\mathrm{C}_{16}-\mathrm{C}_{18}$ ester. 

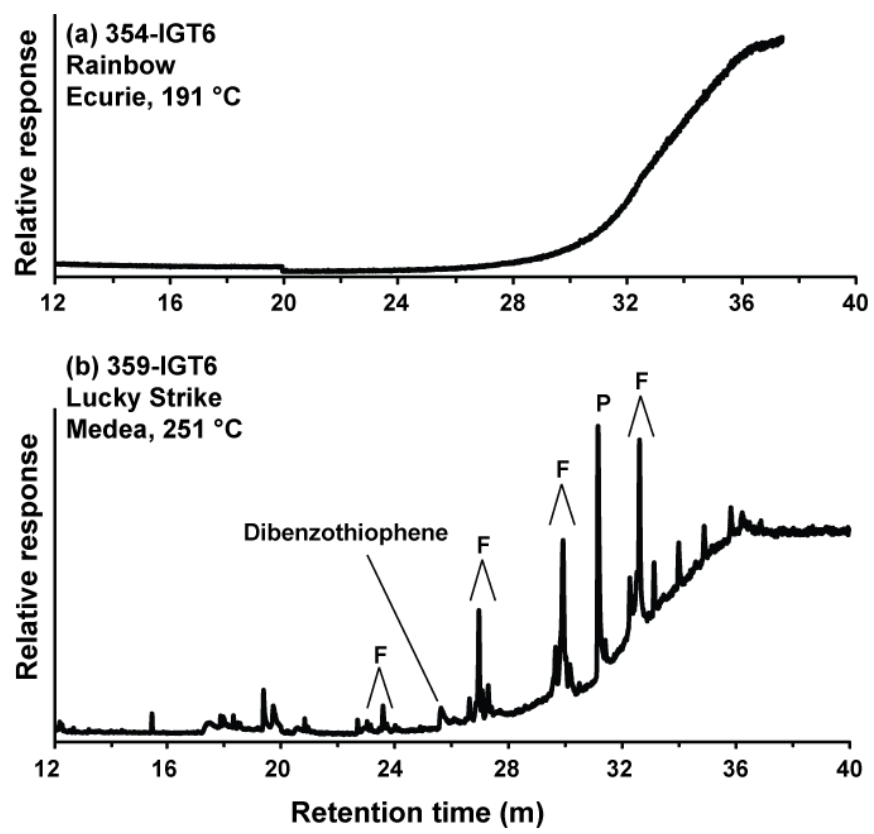

Figure 7. Total ion chromatograms for selected GC-MS analysis of extracts of IGT fluid samples. Families of peaks marked with an "F" represent contamination from the Fluorolube lubricant used in the samplers, and the peak labeled "P" is a phthalate. (a) Extract of fluid from the Ecurie site at Rainbow, showing an absence of peaks above the instrumental baseline. Most other fluid extracts were similar. (b) Extract of fluid from the Medea site at Lucky Strike. Unlabeled peaks in the chromatograms did not match any compounds in the spectral library, and are presumably additional contaminants introduced during sample processing. 

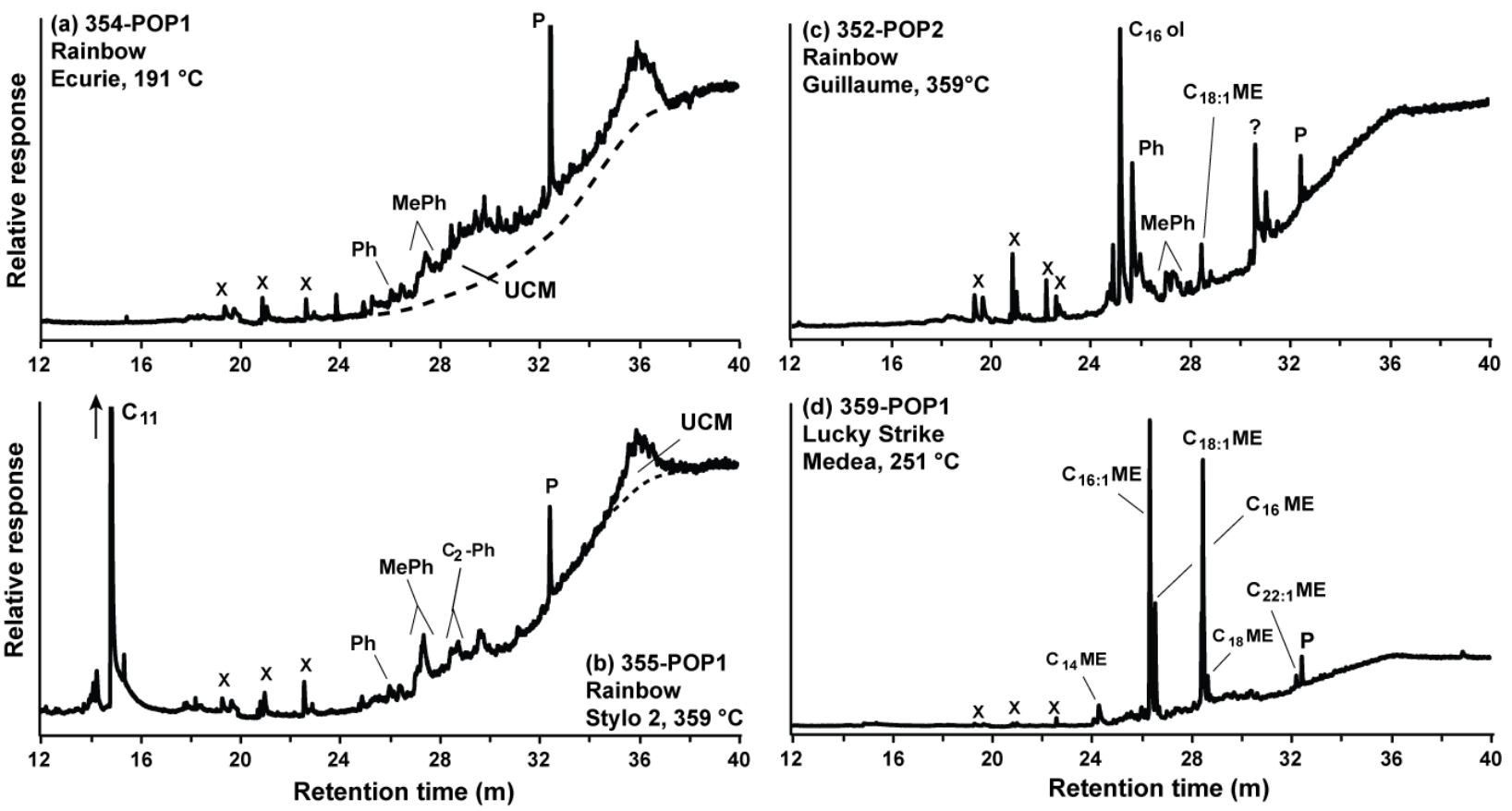

Figure 8. Total ion chromatograms for representative MeOH extracts of POP gun samples. The dashed lines in the chromatograms represent the instrument baseline. (a) Sample from the Ecurie vent at Rainbow $\left(191{ }^{\circ} \mathrm{C}\right.$; J2-354-POP1) showing a small, broad UCM extending from 25 to 38 min and peaks for phenanthrene $(\mathrm{Ph})$ and methylphenanthrenes $(\mathrm{MePh})$. The sample also contains several $\mathrm{C}_{2}$-phenanthrenes $\left(\mathrm{C}_{2}-\mathrm{Ph}\right)$, but peaks for these compounds are obscured by the UCM. The other POP gun sample at this site (J2-354-POP2) produced very similar results. (b) Sample from the Stylo 2 site at Rainbow $\left(359^{\circ} \mathrm{C}\right.$; J2-355-POP1) showing small peaks for several $\mathrm{PAH}$ including $\mathrm{Ph}, \mathrm{MePh}$, and $\mathrm{C}_{2}-\mathrm{Ph}$, as well as a small UCM centered at about $36 \mathrm{~min}$. Extracts for other POP gun samples at the high-temperature Stylo 2 and Padraig sites at Rainbow showed similar trace levels of aromatic compounds. (c) Sample from the $359{ }^{\circ} \mathrm{C}$ Guillaume site at Rainbow. In addition to $\mathrm{Ph}$ and $\mathrm{MePh}$, peaks corresponding to hexadecanol $\left(\mathrm{C}_{16} \mathrm{Ol}\right)$ and octadecenoic acid methyl ester $\left(\mathrm{C}_{18: 1} \mathrm{ME}\right)$ are present. The other POP gun sample at this site produced identical results. (d) Sample from the Medea vent at Lucky Strike $\left(251^{\circ} \mathrm{C}\right)$, including methyl esters of a number of alkanoic and alkenoic acids $\left(\mathrm{C}_{x} \mathrm{ME}\right.$ and $\mathrm{C}_{x: 1} \mathrm{ME}$, respectively, where $x$ is the carbon number). Undecane $\left(\mathrm{C}_{11}\right)$ comes from the methanol solvent. Peaks marked with an " $X$ " were unidentifiable compounds present in all samples and are presumably contaminants introduced during sample processing (see Supplemental Fig. S1). "P" = dioctyl phthalate. 

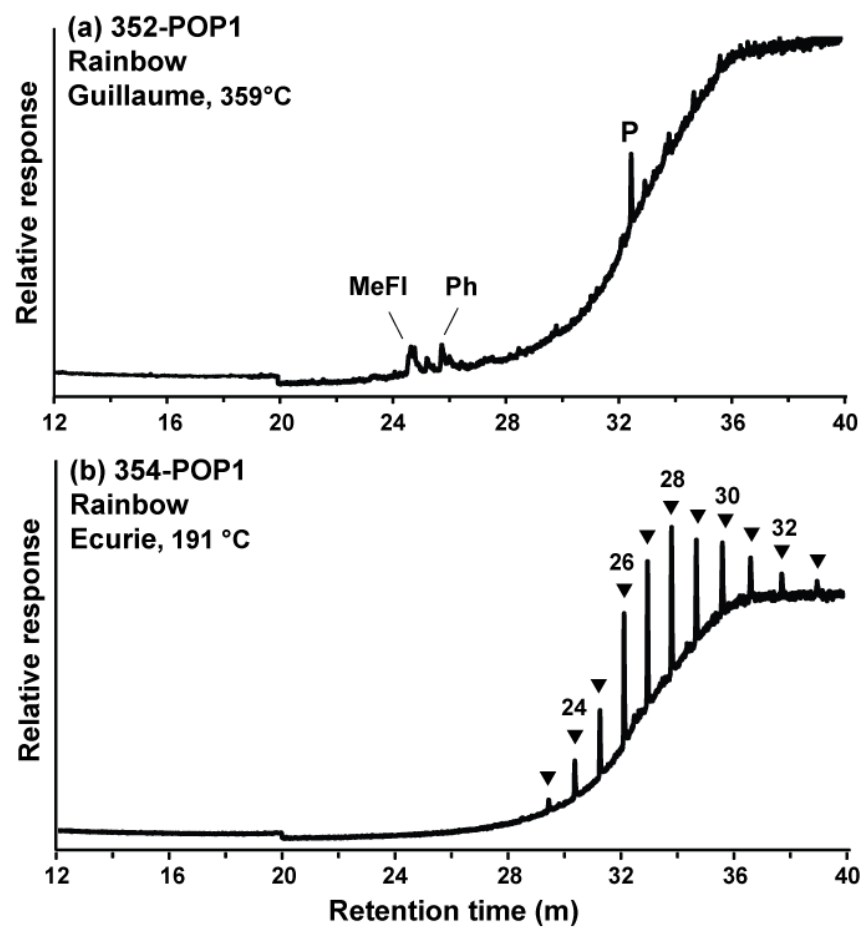

Figure 9. Total ion chromatograms for selected DCM extracts of POP gun samples. (a) Sample from the $359{ }^{\circ} \mathrm{C}$ Guillaume site at Rainbow, showing trace amounts of methylfluorene (MeFl) and phenanthrene $(\mathrm{Ph})$. (b) Sample from the Ecurie site at Rainbow, showing peaks for a homologous series of $n$-alkanes with 23-33 carbon atoms (inverted triangles; labels show carbon chain length). Peak labeled "P" is dioctylphthalate. DCM extracts for other POP gun samples showed no organic compounds attributable to the hydrothermal fluids. 
(a) 354-SPE(HLB) DCM extract - Rainbow (Ecurie)

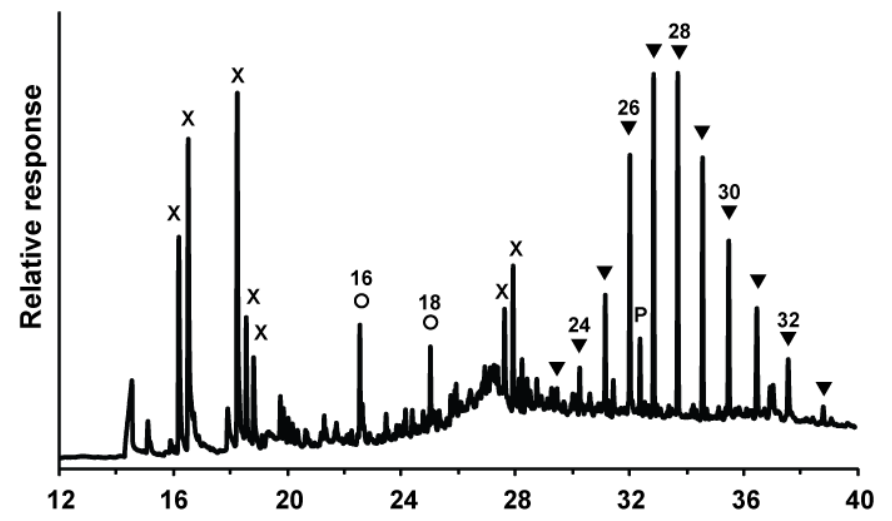

(b) 359-SPE(HLB) DCM extract - Lucky Strike (Medea)

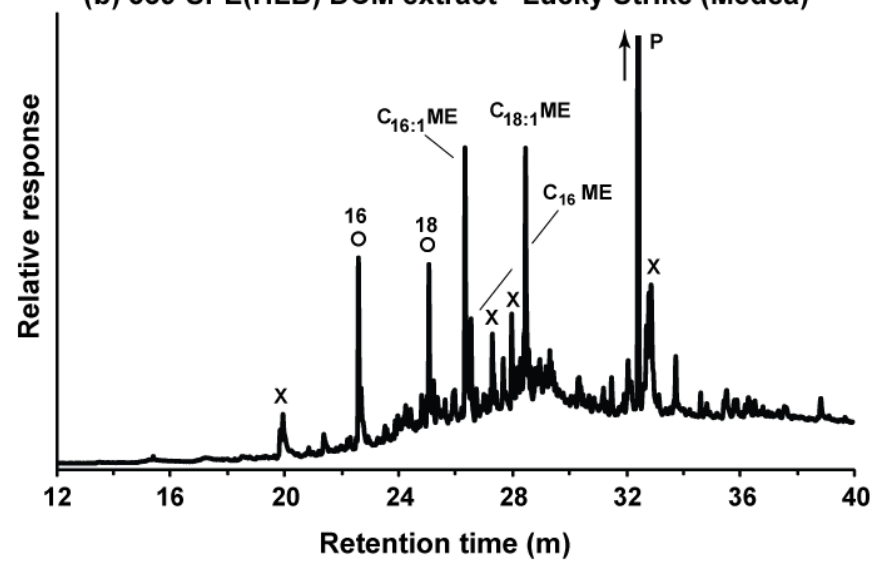

Figure 10. Total ion chromatograms for selected HLB SPE cartridge samples extracted with DCM. (a) Sample from the Ecurie site at Rainbow, showing peaks for a homologous series of $n$ alkanes with 23-33 carbon atoms (inverted triangles; numbers show carbon chain length). (c) Sample from Medea vent at Lucky Strike. Included in the extract are methyl esters of several alkanoic and alkenoic acids $\left(\mathrm{C}_{x} \mathrm{ME}\right.$ and $\mathrm{C}_{x: 1} \mathrm{ME}$, respectively, where $x$ is the carbon number). Prominent peaks labeled with " $X$ " as well as the broad hump of compounds centered at $\sim 27.5 \mathrm{~m}$ are present in all DCM extracts of the HLB cartridges and are inferred to be contaminants from the SPE microbeads (see Supplemental Fig. S1 for examples). Note that the sample shown in (b) was inadvertently allowed to evaporate to dryness, resulting in loss of compounds at $<19 \mathrm{~m}$. Also identified as ubiquitous contaminants from the SPE microbeads are $\mathrm{C}_{16}$ and $\mathrm{C}_{18} n$-alkenes (labeled with open circles). Peak labeled "P" is dioctylphthalte. 


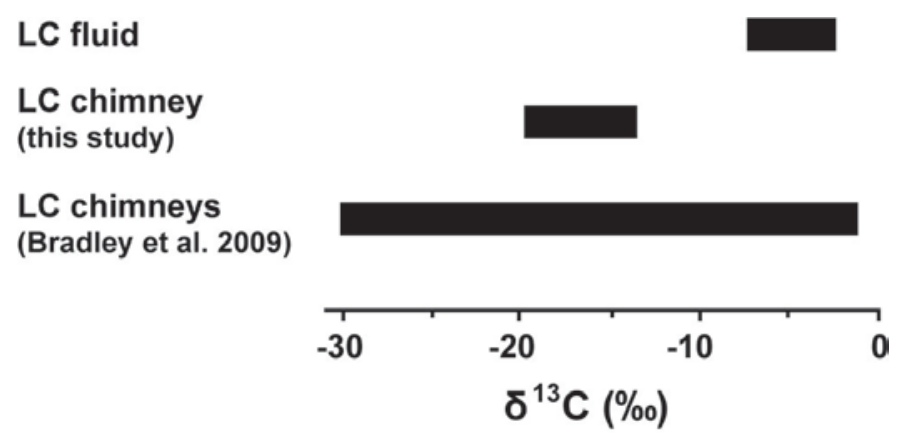

Figure 11. Range of carbon isotopic compositions for $n$-alkanoic acids measured for fluid and chimney samples from Lost City in this study, and reported for multiple active chimney samples from Lost City by Bradley et al. (2009). 


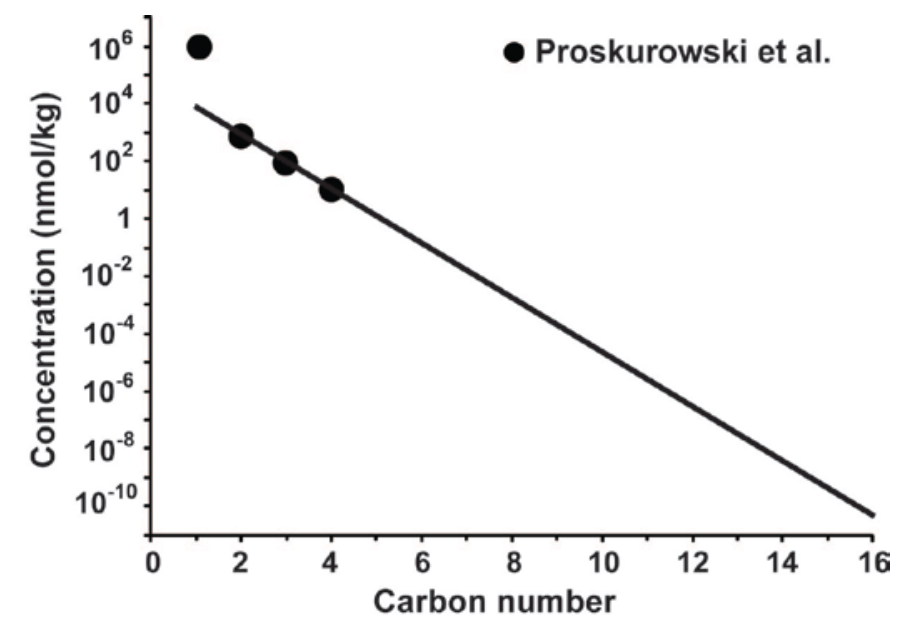

Figure 12. Predicted concentrations of linear alkanes as function of carbon number (line) based on extrapolation of measurements of $\mathrm{C}_{2}-\mathrm{C}_{4}$ alkanes measured at the Beehive vent at Lost City by Proskurowski et al. (2008) (circles). Line calculated assuming a log-linear decrease in alkane abundance with increasing carbon number typical of Fischer-Tropsch-type organic synthesis reactions. 